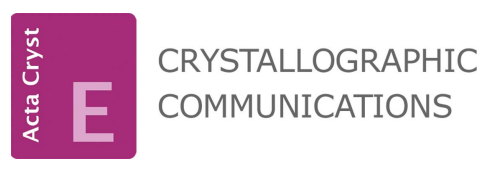

ISSN 2056-9890
Received 26 July 2018

Accepted 30 July 2018

Edited by M. Zeller, Purdue University, USA

Keywords: crystal structure; NiFe hydrogenase; enzyme model; bioinorganic; sulfur ligand.

CCDC reference: 1859284

Supporting information: this article has supporting information at journals.iucr.org/e

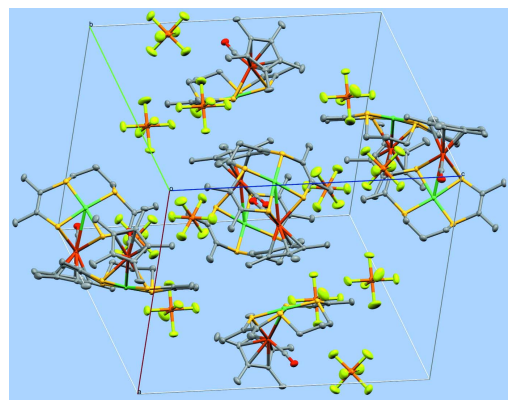

OPEN $\odot$ ACCESS

\section{A new structural model for NiFe hydrogenases: an unsaturated analogue of a classic hydrogenase model leads to more enzyme-like $\mathrm{Ni}-$ Fe distance and interplanar fold}

\author{
Daniel J. Harrison, ${ }^{a}$ Alan J. Lough ${ }^{\mathrm{b}}$ and Ulrich Fekl ${ }^{\mathrm{a} *}$
}

${ }^{\mathbf{a}}$ Department of Chemical and Physical Sciences, University of Toronto Mississauga, 3359 Mississauga Rd, Mississauga,
Ontario, L5L 1C6, Canada, and ${ }^{\mathbf{b}}$ Department of Chemistry, University of Toronto, 80 St. George Street, Toronto, Ontario,
M5S 3H6, Canada. *Correspondence e-mail: ulrich.fekl@utoronto.ca

The complex cation in the title compound, (carbonyl- $1 \kappa C)\left(1 \eta^{5}\right.$-pentamethylcyclopentadienyl)( $\mu$-2,3,9,10-tetramethyl-1,4,8,11-tetrathiaundeca-2,9-diene1,11-diido- $\left.1 \kappa^{2} S, S^{\prime \prime \prime}: 2 \kappa^{4} S, S^{\prime}, S^{\prime \prime}, S^{\prime \prime \prime}\right)$ ironnickel $(\mathrm{Fe}-\mathrm{Ni})$ hexafluorophosphate, [Fe$\left.\mathrm{Ni}\left(\mathrm{C}_{10} \mathrm{H}_{15}\right)\left(\mathrm{C}_{11} \mathrm{H}_{18} \mathrm{~S}_{4}\right)(\mathrm{CO})\right] \mathrm{PF}_{6}$ or $\left[\mathrm{Ni}\left(L^{\prime}\right) \mathrm{FeCp} *(\mathrm{CO})\right] \mathrm{PF}_{6}$, is composed of the nickel complex fragment $\left[\mathrm{Ni}\left(L^{\prime}\right)\right]$ coordinated as a metalloligand (using $\mathrm{S}^{1}$ and $\left.\mathrm{S}^{4}\right)$ to the $[\mathrm{FeCp} *(\mathrm{CO})]^{+}$fragment, where $\left(L^{\prime}\right)^{2-}$ is $[\mathrm{S}-\mathrm{C}(\mathrm{Me})=\mathrm{C}(\mathrm{Me})-\mathrm{S}-$ $\left.\left(\mathrm{CH}_{2}\right)_{3}-\mathrm{S}-\mathrm{C}(\mathrm{Me})=\mathrm{C}(\mathrm{Me})-\mathrm{S}\right]^{2-}$ and where $\mathrm{Cp}^{*-}$ is cyclo- $\mathrm{C}_{5}(\mathrm{Me})_{5}{ }^{-}$(pentamethylcyclopentadienyl). The ratio of hexafluorophosphate anion per complex cation is $1: 1$. The structure at $150 \mathrm{~K}$ has orthorhombic $(\mathrm{Pbcn})$ symmetry. The atoms of the complex cation are located on general positions (multiplicity $=8$ ), whereas there are two independent hexafluorophosphate anions, each located on a twofold axis (Wyckoff position $4 c$; multiplicity $=4$ ). The structure of the new dimetallic cation $\left[\mathrm{Ni}\left(L^{\prime}\right) \mathrm{FeCp} *(\mathrm{CO})\right]^{+}$can be described as containing a three-legged piano-stool environment for iron $\left[\mathrm{Cp} * \mathrm{Fe}(\mathrm{CO})^{\prime} \mathrm{S}_{2}\right.$ '] and an approximately square-planar ' $\mathrm{S}_{4}$ ' environment for $\mathrm{Ni}$. The $\mathrm{NiS}_{2} \mathrm{Fe}$ diamondshaped substructure is notably folded at the $\mathrm{S}-\mathrm{S}$ hinge: the angle between the $\mathrm{NiS}_{2}$ plane and the $\mathrm{FeS}_{2}$ plane normals is $64.85(6)^{\circ}$. Largely because of this fold, the nickel-iron distance is relatively short, at 2.9195 (8) $\AA$. The structural data for the complex cation, which contains a new unsaturated ' $\mathrm{S}_{4}$ ' ligand (two $\mathrm{C}=\mathrm{C}$ double bonds), provide an interesting comparison with the known $\mathrm{NiFe}$ hydrogenase models containing a saturated ' $\mathrm{S}_{4}$ '-ligand analogue having the same number of carbon atoms in the ligand backbone, namely with the structures of $[\mathrm{Ni}(L) \mathrm{FeCp}(\mathrm{CO})]^{+}$(as the $\mathrm{PF}_{6}{ }^{-}$salt, $\mathrm{CH}_{2} \mathrm{Cl}_{2}$ solvate) and $[\mathrm{Ni}(L) \mathrm{FeCp} *(\mathrm{CO})]^{+}$(as the $\mathrm{PF}_{6}^{-}$salt), where $(L)^{2-}$ is $\left[\mathrm{S}-\mathrm{CH}_{2}-\mathrm{CH}_{2}-\mathrm{S}-\right.$ $\left.\left(\mathrm{CH}_{2}\right)_{3}-\mathrm{S}-\mathrm{CH}_{2}-\mathrm{CH}_{2}-\mathrm{S}\right]^{2-}$ and $\mathrm{Cp}^{-}$is cyclopentadienyl. The saturated analogues $[\mathrm{Ni}(L) \mathrm{FeCp}(\mathrm{CO})]^{+}$and $[\mathrm{Ni}(L) \mathrm{FeCp} *(\mathrm{CO})]^{+}$have similar $\mathrm{Ni}-\mathrm{Fe}$ distances: 3.1727 (6), 3.1529 (7) $\AA$ (two independent molecules in the unit cell) and $3.111(5) \AA$, respectively, for the two complexes, whereas $\left[\mathrm{Ni}\left(L^{\prime}\right) \mathrm{FeCp} *(\mathrm{CO})\right]^{+}$described here stands out with a much shorter $\mathrm{Ni}-\mathrm{Fe}$ distance $[2.9196(8) \AA]$. Also, $[\mathrm{Ni}(L) \mathrm{FeCp}(\mathrm{CO})]^{+}$and $[\mathrm{Ni}(L) \mathrm{FeCp} *(\mathrm{CO})]^{+}$show interplanar fold angles that are similar between the two: $39.56(5), 41.99$ (5) (independent molecules in the unit cell) and $47.22(9)^{\circ}$, respectively, whereas $\left[\mathrm{Ni}\left(L^{\prime}\right) \mathrm{FeCp} *(\mathrm{CO})\right]^{+}$possesses a much more pronounced fold $\left[64.85(6)^{\circ}\right]$. Given that larger fold angles and shorter $\mathrm{Ni}-\mathrm{Fe}$ distances are considered to be structurally closer to the enzyme, unsaturation in an ' $\mathrm{S}_{4}$ '-ligand of the type (S$\left.\mathrm{C}_{2}-\mathrm{S}-\mathrm{C}_{3}-\mathrm{S}-\mathrm{C}_{2}-\mathrm{S}\right)^{2-}$ seems to increase structural resemblance to the enzyme for structural models of the type $\left[\mathrm{Ni}\left({ }^{\prime} \mathrm{S}_{4}{ }^{\prime}\right) \mathrm{FeCp}^{R}(\mathrm{CO})\right]^{+}\left(\mathrm{Cp}^{R}=\mathrm{Cp}\right.$ or $\mathrm{Cp} *$.

\section{Chemical context}

Since the discovery and structural elucidation of nickel-iron hydrogenases, synthetic chemists have worked towards closer 


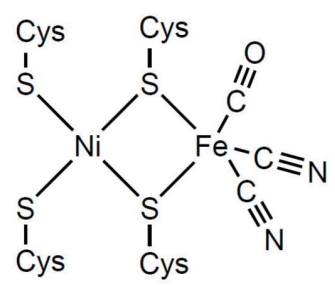

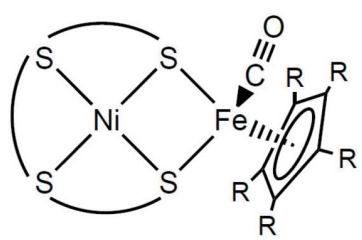

Figure 1

Structure of the NiFe hydrogenase active site (left) and general model of the type $\left[\mathrm{Ni}\left({ }^{\prime} \mathrm{S}_{4} \text { ') } \mathrm{Fe}\left(\mathrm{Cp}^{R}\right)(\mathrm{CO})\right]^{+}\right.$(right; ' $\mathrm{S}_{4}$ ' = synthetic tetrasulfur donor ligand).

and closer structural models for the $\mathrm{NiFe}$ hydrogen-splitting active site (Lubitz et al., 2014). This active site contains two terminal sulfur donors and two bridging sulfur donors coordinated to nickel, as well as a pseudo-octahedal coordination sphere around iron, which is completed by cyano and carbonyl ligands (Fig. 1, left). Several closely related models of the active site have been prepared by combining an $\mathrm{Ni}\left(\right.$ ' $\mathrm{S}_{4}$ ') fragment (' $\mathrm{S}_{4}{ }^{\prime}=$ dianionic tetradentate sulfur ligand) with an $\left[\mathrm{FeCp}^{R}(\mathrm{CO})\right]^{+}$fragment $\left(\mathrm{Cp}^{R}=\mathrm{Cp}, \mathrm{C}_{5} \mathrm{H}_{5}\right.$ or $\left.\mathrm{Cp}^{*}, \mathrm{C}_{5} \mathrm{Me}_{5}\right)$, as illustrated in Fig. 1 (right) (Canaguier et al., 2010; Yang et al., 2015; Zhu et al., 2005). These complexes have an overall mono-cationic charge, consistent with formal $\mathrm{Ni}^{\mathrm{II}}$ and $\mathrm{Fe}^{\mathrm{II}}$ oxidations states. The first ' $\mathrm{S}_{4}$ ' ligand used in this capacity featured a saturated two-three-two carbon linker, in $L^{2-}=$ $\left[\mathrm{S}-\mathrm{CH}_{2}-\mathrm{CH}_{2}-\mathrm{S}-\left(\mathrm{CH}_{2}\right)_{3}-\mathrm{S}-\mathrm{CH}_{2}-\mathrm{CH}_{2}-\mathrm{S}\right]^{2-}$ (Fig. 2, left) (Yang et al., 2015; Zhu et al., 2005).

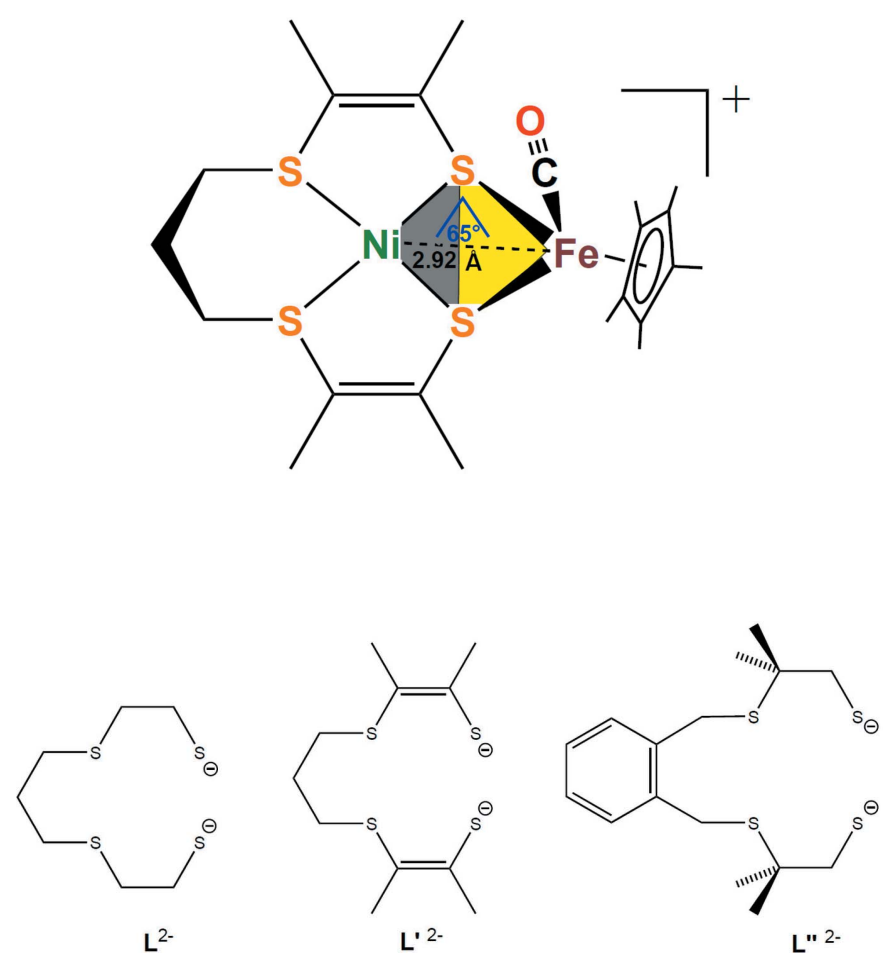

Figure 2

' $\mathrm{S}_{4}$ ' ligands used for the structurally characterized NiFe hydrogenase models of the type $\left[\mathrm{Ni}\left({ }^{(} \mathrm{S}_{4}{ }^{\prime}\right) \mathrm{Fe}\left(\mathrm{Cp}^{R}\right)(\mathrm{CO})\right]^{+}$.
Here, we present a new [ $\left.\mathrm{Ni}\left({ }^{\prime} \mathrm{S}_{4}{ }^{\prime}\right) \mathrm{FeCp}^{R}(\mathrm{CO})\right]^{+}$model based on an analogous but unsaturated ' $\mathrm{S}_{4}$ ' ligand, namely $L^{\prime 2-}=$ $\left[\mathrm{S}-\mathrm{C}(\mathrm{Me})=\mathrm{C}(\mathrm{Me})-\mathrm{S}-\left(\mathrm{CH}_{2}\right)_{3}-\mathrm{S}-\mathrm{C}(\mathrm{Me})=\mathrm{C}(\mathrm{Me})-\mathrm{S}\right]^{2-}$ (Fig. 2, middle), and assess the structural consequences of incorporating the unsaturated ligand. For comparison, we will also discuss a literature $\left[\mathrm{Ni}\left({ }^{\prime} \mathrm{S}_{4}\right) \mathrm{Fe}\left(\mathrm{Cp}^{R}\right)(\mathrm{CO})\right]^{+}$complex in which the ' $\mathrm{S}_{4}$ ' ligand has a four-carbon linker in the remote portion of the backbone ( $L^{\prime \prime 2-}$, Fig. 2, right) (Canaguier et al., 2010).

\section{Structural commentary}

$\left[\mathrm{Ni}\left(L^{\prime}\right) \mathrm{FeCp} *(\mathrm{CO})\right]^{+}$was obtained as solvent-free crystals containing the $\mathrm{PF}_{6}{ }^{-}$counter-ion. A drawing showing both cation and anion in this salt is shown below (see Supramolecular features), and the intramolecular structural features of the cation are discussed first. The structure of $\left[\mathrm{Ni}\left(L^{\prime}\right) \mathrm{FeCp} *(\mathrm{CO})\right]^{+}$is shown in Fig. 3. It contains a threelegged piano stool environment for iron and an approximately square-planar ' $\mathrm{S}_{4}$ ' environment for $\mathrm{Ni}$ (sum of bond angles around Ni1 $=359.83^{\circ}$ ). Selected metal-ligand distances are $\mathrm{Ni1}-\mathrm{S} 1=2.1616(11), \mathrm{Ni1}-\mathrm{S} 2=2.1530(12), \mathrm{Ni1}-\mathrm{S} 3=$ $2.1507(11), \quad \mathrm{Ni} 1-\mathrm{S} 4=2.1563(12) \AA$, and $\mathrm{Fe} 1-\mathrm{S} 1=$ 2.3309 (12), $\mathrm{Fe} 1-\mathrm{S} 4=2.3602(12), \mathrm{Fe} 1-\mathrm{C} 11=1.768$ (5), $\mathrm{Fe} 1-\mathrm{C} 1=2.080(4), \mathrm{Fe} 1-\mathrm{C} 2=2.107(4), \mathrm{Fe} 1-\mathrm{C} 3=$ 2.126 (4), $\mathrm{Fe} 1-\mathrm{C} 4=2.138$ (4), $\mathrm{Fe} 1-\mathrm{C} 5=2.098$ (4) $\AA$. The intermetallic ( $\mathrm{Ni} 1-\mathrm{Fe} 1)$ distance is relatively short, i.e. 2.9195 (8) $\AA$. The $\mathrm{NiS}_{2} \mathrm{Fe}$ diamond is markedly folded at the $\mathrm{S}-\mathrm{S}$ hinge: the angle between the $\mathrm{NiS}_{2}$ plane and the $\mathrm{FeS}_{2}$ plane normals (dihedral angle; $180^{\circ}$ - hinge angle) is $64.85(6)^{\circ}$, and this fold largely accounts for the short nickeliron distance.

In the following discussion, we compare the structural features obtained with the unsaturated ligand $L^{2-}$ with those

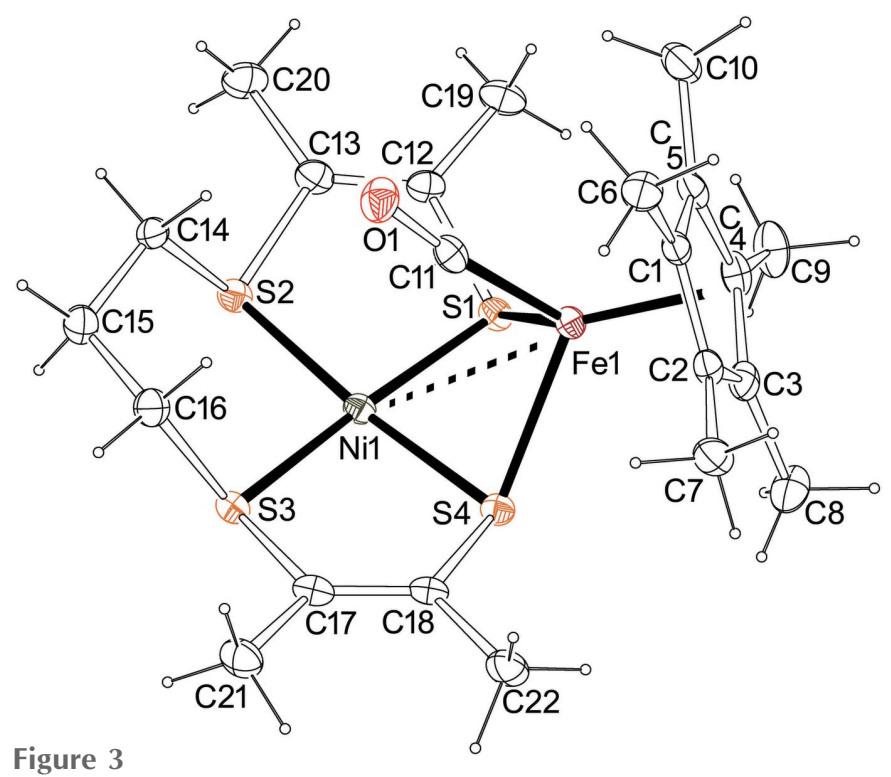

Displacement ellipsoid $\quad(30 \%$ probability $)$ drawing for $\left[\mathrm{Ni}\left(L^{\prime}\right) \mathrm{FeCp} *(\mathrm{CO})\right]^{+}$, as observed in the structure of $\left[\mathrm{Ni}\left(L^{\prime}\right) \mathrm{FeCp} *(\mathrm{CO})\right]\left[\mathrm{PF}_{6}\right]$. Generated using ORTEP-3 for Windows (Farrugia, 2012). 
of literature complexes using the saturated ligand $L^{2-}$. The structures of $[\mathrm{Ni}(L) \mathrm{FeCp}(\mathrm{CO})]^{+}$, as the $\mathrm{PF}_{6}{ }^{-}$salt/ $\mathrm{CH}_{2} \mathrm{Cl}_{2}$ solvate (Zhu et al., 2005), and $[\mathrm{Ni}(L) \mathrm{FeCp} *(\mathrm{CO})]^{+}$, as the $\mathrm{PF}_{6}{ }^{-}$salt (Yang et al., 2015), are known. Both saturated analogues $[\mathrm{Ni}(L) \mathrm{FeCp}(\mathrm{CO})]^{+}$and $[\mathrm{Ni}(L) \mathrm{FeCp} *(\mathrm{CO})]^{+}$show $\mathrm{Ni}-\mathrm{Fe}$ distances that are similar for the two, $3.1727(6) /$ 3.1529 (7) $\AA$ (two independent molecules in the unit cell) and 3.111 (5) $\AA$, respectively, for the two complexes. The $\left[\mathrm{Ni}\left(L^{\prime}\right) \mathrm{FeCp} *(\mathrm{CO})\right]^{+}$complex, on the other hand, has a much shorter $\mathrm{Ni}-\mathrm{Fe}$ distance [2.9195(8), see above]. Also, $[\mathrm{Ni}(L) \mathrm{FeCp}(\mathrm{CO})]^{+}$and $[\mathrm{Ni}(L) \mathrm{FeCp} *(\mathrm{CO})]^{+}$show interplanar fold angles that are similar for the two, $39.56(5) / 41.99(5)^{\circ}$ (two independent molecules in the unit cell) and $47.22(9)^{\circ}$, respectively, while $\left[\mathrm{Ni}\left(L^{\prime}\right) \mathrm{FeCp} *(\mathrm{CO})\right]^{+}$has a much larger fold angle of $64.85(6)^{\circ}$ (see above). The large fold angle and short $\mathrm{Ni}-\mathrm{Fe}$ distance observed in the complex with the unsaturated ligand $L^{\prime}$ match the structure of the enzymatic active site more closely than the angles/distances of the complexes containing the saturated ligand $L$. For eight structurally characterized enzymes, the dihedral angles range from 59 to $99^{\circ}$ and the $\mathrm{Ni}-\mathrm{Fe}$ distances range from 2.53 to $2.97 \AA$ (one outlier being desulfovibrio fructosovorans with $46^{\circ}$ and $3.23 \AA$; Zhu et al., 2005). We have thus provided evidence that unsaturation in an ' $\mathrm{S}_{4}$ '-ligand of the type $\left(\mathrm{S}-\mathrm{C}_{2}-\mathrm{S}-\mathrm{C}_{3}-\mathrm{S}-\mathrm{C}_{2}-\mathrm{S}\right)^{2-}$ can increase structural resemblance to the enzyme in models of the type $\left[\mathrm{Ni}\left({ }^{\prime} \mathrm{S}_{4}{ }^{\prime}\right) \mathrm{FeCp}^{R}(\mathrm{CO})\right]^{+}$. Structural similarity to the enzyme in models was, in alternative approaches, also favoured when additional donor atoms were incorporated into the ligand chain (such as ' $\mathrm{S}_{3} \mathrm{~N}_{2}$ ') or where two bidentate chelate ligands were used instead of one large ' $S_{4}$ ' ligand. (Zhu et al., 2005) Within the context of linear ' $\mathrm{S}_{4}$ ' ligands, an $\left[\mathrm{Ni}\left(L^{\prime \prime}\right) \mathrm{FeCp} *(\mathrm{CO})\right]^{+}$model with four carbon atoms, instead of three, in the remote portion of the backbone (see $L^{\prime \prime 2-}$ in Fig. 2, right) provided an $\mathrm{Ni}-\mathrm{Fe}$ distance and fold angle very similar to those of the $L^{\prime}$ analogue, of 2.9611 (8) $\AA$ and $62.48(4)^{\circ}$, respectively (Canaguier et al., 2010). In terms of activity, $\left[\mathrm{Ni}\left(L^{\prime \prime}\right) \mathrm{FeCp} *(\mathrm{CO})\right]^{+}$was shown to be active as a hydrogen-production catalyst (Canaguier et al., 2010), which suggests that the $\left[\mathrm{Ni}\left(L^{\prime}\right) \mathrm{Cp}^{*}(\mathrm{CO})\right]^{+}$complex, with the unsaturated ' $\mathrm{S}_{4}$ ' ligand $L$ ', might warrant deeper investigation. We conclude that the introduction of unsaturation in the ' $\mathrm{S}_{4}$ ' ligand led to a better structural model relative to the unsaturated ligand, highlighting a new variant of the classic $\left[\mathrm{Ni}\left({ }^{\prime} \mathrm{S}_{4}{ }^{\prime}\right) \mathrm{FeCp}^{R}(\mathrm{CO})\right]^{+}$-type hydrogenase model.

\section{Supramolecular features}

The structure results from packing of discrete cations $\left[\mathrm{Ni}\left(L^{\prime}\right) \mathrm{FeCp} *(\mathrm{CO})\right]^{+}$with hexafluorophosphate anions, without solvent molecules and without any solvent-accessible void. The ratio of hexafluorophosphate anion per complex cation is 1:1. The atoms of the complex cation are situated on general positions (multiplicity $=8$ ), whereas there are two independent hexafluorophosphate anions, each situated on a twofold axis (Wyckoff position $4 c$ in Pbcn; multiplicity =4). A picture of the packing is shown in Fig. 4 (top, 30\% probability ellipsoids), along with labeling of all non-H atoms in the unit cell (bottom). There are no classical hydrogen bonds but there are $\mathrm{C}-\mathrm{H} \cdots \mathrm{F}$ hydrogen bonds to hexafluorophosphate (C6$\mathrm{H} 6 B \cdots \mathrm{F} 4=2.55 \AA ; \quad \mathrm{C} 15-\mathrm{H} 15 B \cdots \mathrm{F}^{\mathrm{i}}=2.55 \AA$ A $\mathrm{C} 21-$ $\left.\mathrm{H} 21 C \cdots \mathrm{F} 4^{\mathrm{ii}}=2.48 \AA ; \mathrm{C} 22-\mathrm{H} 22 C \cdots \mathrm{F} 1^{\mathrm{iii}}=2.52 \AA\right)$ and a $\mathrm{C}-$ $\mathrm{H} \cdots \mathrm{O}$ short contact $(\mathrm{C} 14-\mathrm{H} 14 A \cdots \mathrm{O} 1=2.41 \AA)$ [symmetry codes: (i) $-x+2, y,-z+\frac{3}{2}$; (ii) $-x+1, y,-z+\frac{3}{2}$; (iii) $-x+\frac{3}{2}$, $\left.y+\frac{1}{2}, z\right]$.

\section{Database survey}

The Cambridge Crystallographic Database (version 5.39 including updates up to February 2018; Groom et al., 2016) was surveyed. A search was performed aimed at finding $\mathrm{Ni}_{1} \mathrm{Fe}_{1}$ complexes that contain at least one (possibly substituted) cyclopentadienyl unit, at least one carbonyl (CO) coordinated to iron, and a nickel center bonded to at least four sulfurs. The substructure that was used for the search contained a cyclo- $\mathrm{C}_{5}$ unit (any type of bond allowed), a nickel atom bonded to four sulfur atoms (any type of bond allowed), as well as an $\mathrm{Fe}-\mathrm{C}-\mathrm{O}$ unit (any type of bond for $\mathrm{Fe}-\mathrm{C}$ and for $\mathrm{C}-\mathrm{O}$ ). Out of the six hits, RULQEV, RULQOF and RULQUL are trimetallic (instead of dimetallic) complexes (and also do not contain a cyclopentadienyl but rather a saturated five-membered ring within a polycyclic structure). Since they are not very close analogues of $\left[\mathrm{Ni}\left(L^{\prime}\right) \mathrm{FeCp} *(\mathrm{CO})\right]^{+}$, they are not discussed further. LAZVUE (Zhu et al., 2005) contains $[\mathrm{Ni}(L) \mathrm{FeCp}(\mathrm{CO})]^{+}$(as the $\mathrm{PF}_{6}^{-}$salt, $\mathrm{CH}_{2} \mathrm{Cl}_{2}$ solvate), MUDXOA (Yang et al., 2015) contains $\left[\mathrm{Ni}(L) \mathrm{FeCp}^{*}(\mathrm{CO})\right]^{+}$ (as the $\mathrm{PF}_{6}{ }^{-}$salt), and SUWWAJ (Canaguier et al., 2010) contains $\left[\mathrm{Ni}\left(L^{\prime \prime}\right) \mathrm{FeCp} *(\mathrm{CO})\right]^{+}$(as the $\mathrm{BF}_{4}^{-}$salt, $\mathrm{CH}_{2} \mathrm{Cl}_{2}$ solvate). These three complex cations are discussed in detail above.

\section{Synthesis and crystallization}

The syntheses were performed in dried solvents under an inert atmosphere (nitrogen or argon; vacuum) using standard glovebox (MBraun) and Schlenk techniques. Deuterated NMR solvents were from Cambridge Isotopes. $\left[\mathrm{Cp} * \mathrm{Fe}(\mathrm{CO})_{2}\right]_{2}$ was acquired from Alfa Aesar. All other chemicals were obtained from Sigma-Aldrich. Photolysis was performed using a $160 \mathrm{~W}$ mercury vapour lamp (model: Westron Mega-Ray SelfBallasted Zoologist).

$\mathrm{Ni}\left(\mathrm{S}_{2} \mathrm{C}_{2} \mathrm{Me}_{2}\right)_{2}$ : This precursor for the nickel part of the complex was prepared as described in the literature (Schrauzer \& Mayweg, 1965).

$\mathrm{Ni}\left(L^{\prime}\right)$ : $\mathrm{Ni}\left(L^{\prime}\right)$, i.e. $\mathrm{Ni}\left(\mathrm{S}-\mathrm{C}(\mathrm{Me})=\mathrm{C}(\mathrm{Me})-\mathrm{S}-\left(\mathrm{CH}_{2}\right)_{3}-\right.$ $\mathrm{S}-\mathrm{C}(\mathrm{Me})=\mathrm{C}(\mathrm{Me})-\mathrm{S})$ was prepared by alkylation of $\mathrm{Na}_{2}\left[\mathrm{Ni}\left(\mathrm{S}_{2} \mathrm{C}_{2} \mathrm{Me}_{2}\right)_{2}\right]$ using 1,3-dibromopropane. $\mathrm{Na}_{2}\left[\mathrm{Ni}\left(\mathrm{S}_{2} \mathrm{C}_{2}-\right.\right.$ $\left.\mathrm{Me}_{2}\right)$ ] was prepared from $\mathrm{Ni}\left(\mathrm{S}_{2} \mathrm{C}_{2} \mathrm{Me}_{2}\right)_{2}$ by reduction with excess sodium in THF ( $344 \mathrm{~K}, 18 \mathrm{~h}$, in sealed vessel), until the colour had changed from deep purple to brown-yellow. The subsequent alkylation of $\left[\mathrm{Ni}\left(\mathrm{S}_{2} \mathrm{C}_{2} \mathrm{Me}_{2}\right)\right]^{2-}$ using 1,3-dibromopropane was performed analogously to the procedure described by Schrauzer and co-workers for the closely related $\mathrm{Ni}\left(\mathrm{S}-\mathrm{C}(\mathrm{Ph})=\mathrm{C}(\mathrm{Ph})-\mathrm{S}-\left(\mathrm{CH}_{2}\right)_{3}-\mathrm{S}-\mathrm{C}(\mathrm{Ph})=\mathrm{C}(\mathrm{Ph})-\mathrm{S}\right)$.

(Zhang et al., 1992) 


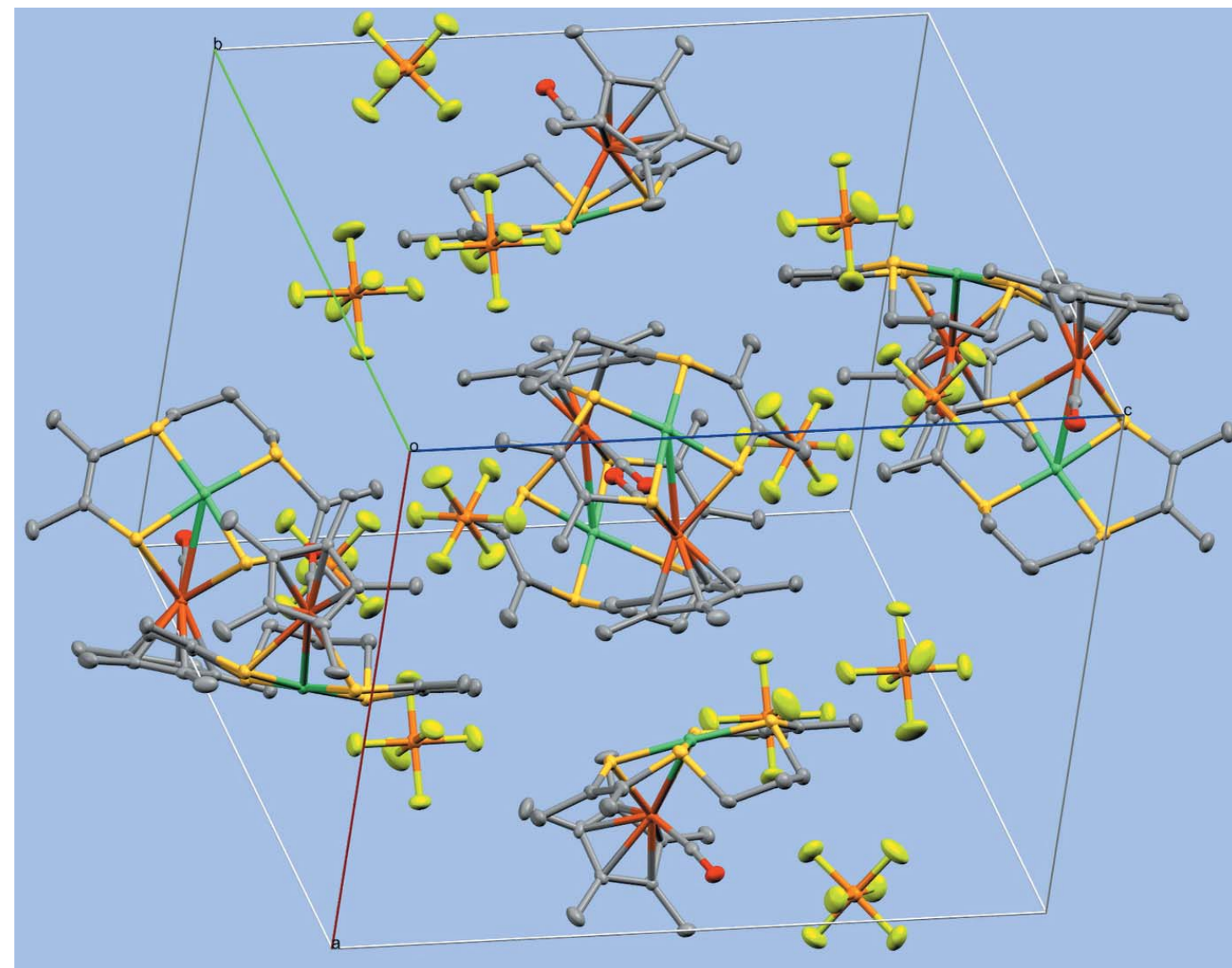

Figure 4

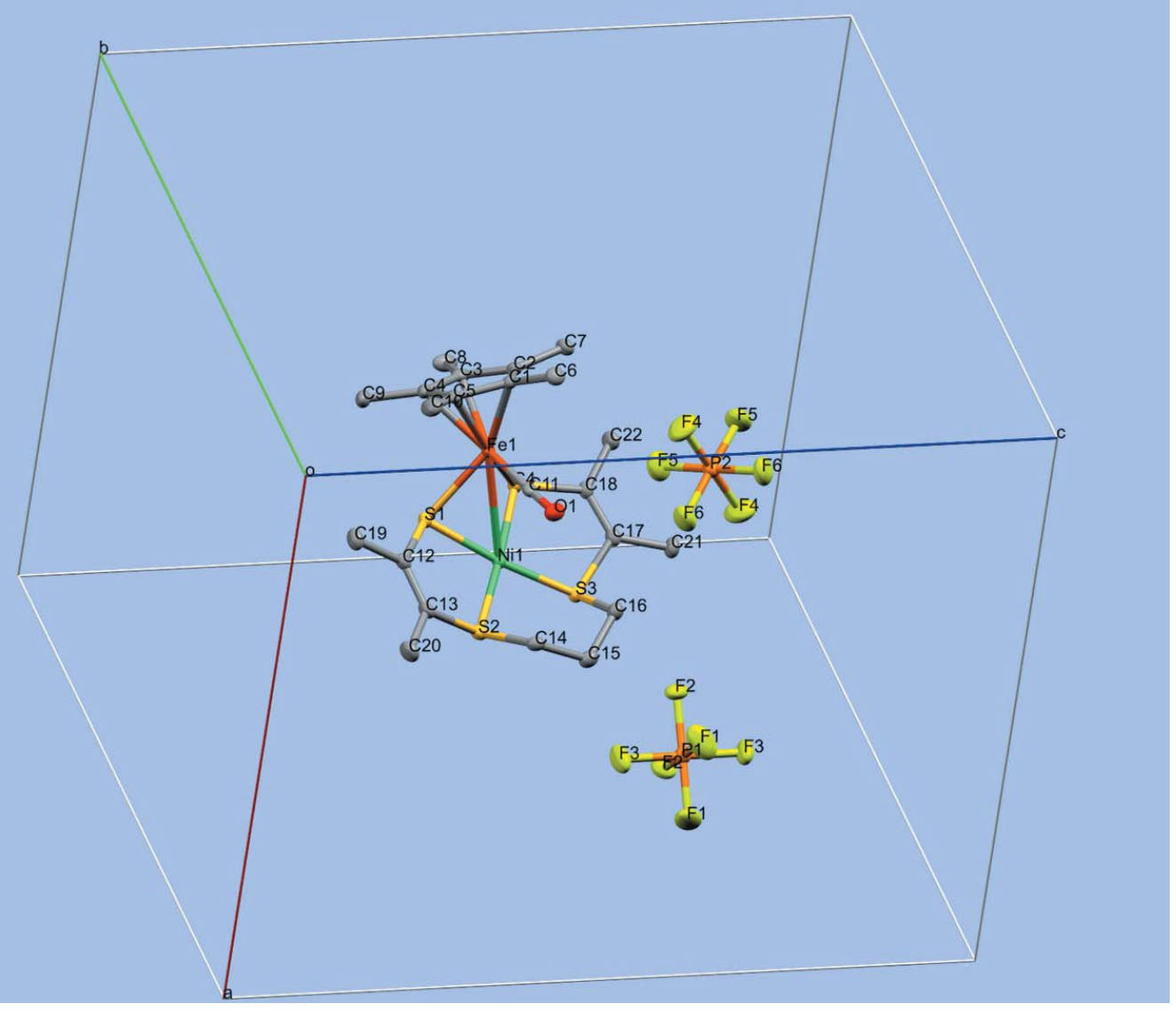

Drawings for packing (top) and labeling (bottom) of all non- $\mathrm{H}$ atoms in $\left[\mathrm{Ni}\left(L^{\prime}\right) \mathrm{FeCp} *(\mathrm{CO})\right]\left[\mathrm{PF}_{6}\right]$. Generated using Mercury (Macrae et al., 2006). For the anion in the bottom part, generic atom labels without symmetry codes have been used. 
Table 1

Experimental details.

\begin{tabular}{|c|c|}
\hline \multicolumn{2}{|l|}{ Crystal data } \\
\hline Chemical formula & {$\left[\mathrm{FeNi}\left(\mathrm{C}_{10} \mathrm{H}_{15}\right)\left(\mathrm{C}_{11} \mathrm{H}_{18} \mathrm{~S}_{4}\right)(\mathrm{CO})\right] \mathrm{PF}_{6}$} \\
\hline$M_{\mathrm{r}}$ & 701.25 \\
\hline Crystal system, space group & Orthorhombic, $\mathrm{Pbcn}$ \\
\hline Temperature $(\mathrm{K})$ & 150 \\
\hline$a, b, c(\AA)$ & $\begin{array}{l}15.4081(3), 18.3762(3), \\
19.2154(3)\end{array}$ \\
\hline$V\left(\AA^{3}\right)$ & $5440.69(16)$ \\
\hline$Z$ & 8 \\
\hline Radiation type & Мо $K \alpha$ \\
\hline$\mu\left(\mathrm{mm}^{-1}\right)$ & 1.65 \\
\hline Crystal size $(\mathrm{mm})$ & $0.20 \times 0.18 \times 0.12$ \\
\hline \multicolumn{2}{|l|}{ Data collection } \\
\hline Diffractometer & Nonius KappaCCD \\
\hline Absorption correction & $\begin{array}{l}\text { Multi-scan (SORTAV; Blessing, } \\
\text { 1995) }\end{array}$ \\
\hline$T_{\min }, T_{\max }$ & $0.759,0.850$ \\
\hline $\begin{array}{l}\text { No. of measured, independent and } \\
\text { observed }[I>2 \sigma(I)] \text { reflections }\end{array}$ & $38285,6224,3874$ \\
\hline$R_{\text {int }}$ & 0.079 \\
\hline$(\sin \theta / \lambda)_{\max }\left(\AA^{-1}\right)$ & 0.649 \\
\hline \multicolumn{2}{|l|}{ Refinement } \\
\hline$R\left[F^{2}>2 \sigma\left(F^{2}\right)\right], w R\left(F^{2}\right), S$ & $0.052,0.148,1.07$ \\
\hline No. of reflections & 6224 \\
\hline No. of parameters & 335 \\
\hline $\mathrm{H}$-atom treatment & $\mathrm{H}$-atom parameters constrained \\
\hline$\Delta \rho_{\max }, \Delta \rho_{\min }\left(\mathrm{e} \AA^{-3}\right)$ & $1.12,-0.73$ \\
\hline
\end{tabular}

Computer programs: COLLECT (Nonius, 1998), DENZO-SMN (Otwinowski \& Minor, 1997), SHELXS97 and SHELXTL (Sheldrick, 2008), SHELXL2016 (Sheldrick, 2015) and PLATON (Spek, 2009)

$\left[\mathrm{Cp} * \mathrm{Fe}(\mathrm{CO})_{2}(\mathrm{NCMe})\right]\left[\mathrm{PF}_{6}\right]$ : This precursor for the iron part of the complex was prepared according to the general procedure for $\left[\mathrm{Cp} * \mathrm{Fe}(\mathrm{CO})_{2}(\text { solvent })\right]^{+}$given by Catheline \& Astruc (1984), using MeCN (acetontrile) as the solvent.

$\left[\mathrm{Ni}\left(L^{\prime}\right) \mathrm{FeCp} *(\mathrm{CO} / \mathrm{NCMe})\right]\left[\mathrm{PF}_{6}\right]: \quad$ Crude $\quad\left[\mathrm{Cp} * \mathrm{Fe}(\mathrm{CO})_{2^{-}}\right.$ $(\mathrm{NCMe})]\left[\mathrm{PF}_{6}\right](210 \mathrm{mg}, 0.48 \mathrm{mmol})$ was combined with $6 \mathrm{ml}$ of acetonitrile and filtered through a glass filter frit. While purging with argon, the reaction was irradiated with UVvisible light $(160 \mathrm{~W}$, see above) for $16 \mathrm{~h}$. Under an inert atmosphere, a solution of $155 \mathrm{mg}(0.46 \mathrm{mmol})$ of $\mathrm{Ni}\left(L^{\prime}\right)$ in $c a$ $7 \mathrm{ml}$ of dichloromethane was added. The reaction mixture was heated under active argon flow to $325 \mathrm{~K}$ for $2 \mathrm{~h}$. After cooling to room temperature, the volatiles were slowly removed under vacuum. The solid was dried under vacuum and stored in the glove-box. Yield of crude product: $253 \mathrm{mg}(75 \%) .{ }^{1} \mathrm{H}$ NMR $\left(200 \mathrm{MHz}, 298 \mathrm{~K}, \mathrm{CD}_{3} \mathrm{CN}\right) \delta 1.60\left[s,\left(\mathbf{C H}_{3}\right)_{5} \mathrm{C}_{5}\right] ; \delta 1.91(s$, $\left.\mathbf{C H}_{3}-\mathrm{C}-\mathrm{S}\right) ; \delta 1.96$ (s, $\left.\mathbf{C H}_{3}-\mathrm{C}-\mathrm{S}\right) ; \delta 2.31\left(s, b r, \mathbf{C H}_{3} \mathrm{CN}-\right.$ $\mathrm{Fe}) ; \delta 2.0-3.7\left[\mathrm{~m}, \mathrm{br}, \mathrm{S}-\left(\mathbf{C H}_{2}\right)_{3}-\mathrm{S}\right]$. Note that the sample thus prepared showed a ${ }^{1} \mathrm{H}$ NMR signal for metal-coordinated acetonitrile. The purpose of the prolonged photolysis was to remove all $\mathrm{CO}$ from iron, in order to selectively prepare $\left[\mathrm{Ni}\left(L^{\prime}\right) \mathrm{FeCp} *(\mathrm{NCMe})\right]\left[\mathrm{PF}_{6}\right]$. However, the sample obtained appeared to be a mixture of $\left[\mathrm{Ni}\left(L^{\prime}\right) \mathrm{FeCp} *(\mathrm{CO})\right]\left[\mathrm{PF}_{6}\right]$ and $\left[\mathrm{Ni}\left(L^{\prime}\right) \mathrm{FeCp} *(\mathrm{NCMe})\right]\left[\mathrm{PF}_{6}\right]$ and is thus referred to as
$\left[\mathrm{Ni}\left(L^{\prime}\right) \mathrm{FeCp} *(\mathrm{CO} / \mathrm{NCMe})\right]\left[\mathrm{PF}_{6}\right]$. Yet, crystallization from acetone yielded exclusively $\left[\mathrm{Ni}\left(L^{\prime}\right) \mathrm{FeCp} *(\mathrm{CO})\right]\left[\mathrm{PF}_{6}\right]$, in crystalline form.

Crystallization of $\left[\mathrm{Ni}\left(L^{\prime}\right) \mathrm{FeCp} *(\mathrm{CO})\right]\left[\mathrm{PF}_{6}\right]: 11 \mathrm{mg}$ of $\left[\mathrm{Ni}\left(L^{\prime}\right) \mathrm{FeCp} *(\mathrm{CO} / \mathrm{NCMe})\right]\left[\mathrm{PF}_{6}\right]$ were dissolved in $1.5 \mathrm{ml}$ of acetone and filtered through $1 \mathrm{~cm}$ of Celite. Through solvent vapor diffusion, by placing the loosely capped vial into a larger vessel containing diethyl ether vapour (and some liquid), crystals of $\left[\mathrm{Ni}\left(L^{\prime}\right) \mathrm{FeCp} *(\mathrm{CO})\right]\left[\mathrm{PF}_{6}\right]$ were grown within two days at $308 \mathrm{~K}$.

\section{Refinement}

Crystal data, data collection and structure refinement details are summarized in Table 1 . All $\mathrm{H}$ atoms were placed in calculated positions and included in the refinment in a ridingmodel approximation with $\mathrm{C}-\mathrm{H}$ distances of 0.98 and $0.99 \AA$ and $U_{\text {iso }}(\mathrm{H})=1.2 U_{\text {eq }}(\mathrm{C})$ or $1.5 U_{\text {eq }}\left(\mathrm{C}_{\text {methyl }}\right)$.

\section{Acknowledgements}

We thank Mitchell J. Kerr for preparing a sample of $\mathrm{Ni}\left(\mathrm{S}_{2} \mathrm{C}_{2} \mathrm{Me}_{2}\right)_{2}$ used in the synthesis.

\section{Funding information}

Funding for this research was provided by: Natural Sciences and Engineering Research Council of Canada; University of Toronto.

\section{References}

Blessing, R. H. (1995). Acta Cryst. A51, 33-38.

Canaguier, S., Field, M., Oudart, Y., Pécaut, J., Fontecave, M. \& Artero, V. (2010). Chem. Commun. 46, 5876-5878.

Catheline, D. \& Astruc, D. (1984). Organometallics, 3, 1094-1100.

Farrugia, L. J. (2012). J. Appl. Cryst. 45, 849-854.

Groom, C. R., Bruno, I. J., Lightfoot, M. P. \& Ward, S. C. (2016). Acta Cryst. B72, 171-179.

Lubitz, W., Ogata, H., Rüdiger, O. \& Reijerse, E. (2014). Chem. Rev. 114, 4081-4148.

Macrae, C. F., Edgington, P. R., McCabe, P., Pidcock, E., Shields, G. P., Taylor, R., Towler, M. \& van de Streek, J. (2006). J. Appl. Cryst. 39, 453-457.

Nonius (1998). COLLECT. Nonius BV, Delft, The Netherlands.

Otwinowski, Z. \& Minor, W. (1997). Methods in Enzymology, Vol. 276, Macromolecular Crystallography, Part A, edited by C. W. Carter Jr \& R. M. Sweet, pp. 307-326. New York: Academic Press.

Schrauzer, G. N. \& Mayweg, V. P. (1965). J. Am. Chem. Soc. 87, $1483-$ 1489.

Sheldrick, G. M. (2008). Acta Cryst. A64, 112-122.

Sheldrick, G. M. (2015). Acta Cryst. C71, 3-8.

Spek, A. L. (2009). Acta Cryst. D65, 148-155.

Yang, D., Li, Y., Su, L., Wang, B. \& Qu, J. (2015). Eur. J. Inorg. Chem. pp. 2965-2973.

Zhang, C., Reddy, H. K., Chadha, R. K. \& Schrauzer, G. N. (1992). J. Coord. Chem. 26, 117-126.

Zhu, W., Marr, A. C., Wang, Q., Neese, F., Spencer, D. J. E., Blake, A. J., Cooke, P. A., Wilson, C. \& Schröder, M. (2005). Proc. Natl Acad. Sci. USA, 102, 18280-18285. 


\section{supporting information}

Acta Cryst. (2018). E74, 1222-1226 [https://doi.org/10.1107/S2056989018010939]

\section{A new structural model for NiFe hydrogenases: an unsaturated analogue of a classic hydrogenase model leads to more enzyme-like $\mathrm{Ni}-\mathrm{Fe}$ distance and interplanar fold}

\section{Daniel J. Harrison, Alan J. Lough and Ulrich Fekl}

Computing details

Data collection: COLLECT (Nonius, 1998); cell refinement: DENZO-SMN (Otwinowski \& Minor, 1997); data reduction: DENZO-SMN (Otwinowski \& Minor, 1997); program(s) used to solve structure: SHELXS97 (Sheldrick, 2008); program(s) used to refine structure: SHELXL2016 (Sheldrick, 2015); molecular graphics: PLATON (Spek, 2009); software used to prepare material for publication: SHELXTL (Sheldrick, 2008).

(Carbonyl-1 $\left.\kappa_{C}\right)\left(1 \eta^{5}\right.$-pentamethylcyclopentadienyl) $(\mu$-2,3,9,10-tetramethyl-1,4,8,11-tetrathiaundeca-2,9diene-1,11-diido-1 $\left.\kappa^{2} S, S^{\prime \prime \prime}: 2 \kappa^{4} S, S^{\prime}, S^{\prime \prime}, S^{\prime \prime \prime}\right)$ ironnickel $(\mathrm{Fe}-\mathrm{Ni})$ hexafluorophosphate

\section{Crystal data}

$\left[\mathrm{FeNi}\left(\mathrm{C}_{10} \mathrm{H}_{15}\right)\left(\mathrm{C}_{11} \mathrm{H}_{18} \mathrm{~S}_{4}\right)(\mathrm{CO})\right] \mathrm{PF}_{6}$

$M_{r}=701.25$

Orthorhombic, $\mathrm{Pbcn}$

$a=15.4081(3) \AA$

$b=18.3762(3) \AA$

$c=19.2154(3) \AA$

$V=5440.69(16) \AA^{3}$

$Z=8$

$F(000)=2880$

Data collection

Nonius KappaCCD diffractometer

Radiation source: fine-focus sealed tube Detector resolution: 9 pixels $\mathrm{mm}^{-1}$ $\varphi$ scans and $\omega$ scans with $\kappa$ offsets Absorption correction: multi-scan (SORTAV; Blessing, 1995)

$T_{\text {min }}=0.759, T_{\max }=0.850$

Refinement

Refinement on $F^{2}$ Least-squares matrix: full $R\left[F^{2}>2 \sigma\left(F^{2}\right)\right]=0.052$ $w R\left(F^{2}\right)=0.148$

$S=1.07$ 6224 reflections
$D_{\mathrm{x}}=1.712 \mathrm{Mg} \mathrm{m}^{-3}$

Mo $K \alpha$ radiation, $\lambda=0.71073 \AA$

Cell parameters from 38285 reflections

$\theta=2.6-27.5^{\circ}$

$\mu=1.65 \mathrm{~mm}^{-1}$

$T=150 \mathrm{~K}$

Block, green

$0.20 \times 0.18 \times 0.12 \mathrm{~mm}$

38285 measured reflections

6224 independent reflections

3874 reflections with $I>2 \sigma(I)$

$R_{\text {int }}=0.079$

$\theta_{\max }=27.5^{\circ}, \theta_{\min }=2.6^{\circ}$

$h=-19 \rightarrow 19$

$k=-23 \rightarrow 23$

$l=-24 \rightarrow 24$

335 parameters

0 restraints

Hydrogen site location: inferred from neighbouring sites

$\mathrm{H}$-atom parameters constrained 
$w=1 /\left[\sigma^{2}\left(F_{\mathrm{o}}^{2}\right)+(0.0765 P)^{2}+2.0266 P\right]$

where $P=\left(F_{\mathrm{o}}^{2}+2 F_{\mathrm{c}}^{2}\right) / 3$

$(\Delta / \sigma)_{\max }=0.002$

$$
\Delta \rho_{\max }=1.12 \mathrm{e} \AA^{-3}
$$

\section{Special details}

Geometry. All esds (except the esd in the dihedral angle between two 1.s. planes) are estimated using the full covariance matrix. The cell esds are taken into account individually in the estimation of esds in distances, angles and torsion angles; correlations between esds in cell parameters are only used when they are defined by crystal symmetry. An approximate (isotropic) treatment of cell esds is used for estimating esds involving 1.s. planes.

Fractional atomic coordinates and isotropic or equivalent isotropic displacement parameters $\left(\AA^{2}\right)$

\begin{tabular}{|c|c|c|c|c|}
\hline & $x$ & $y$ & $z$ & $U_{\text {iso }} * / U_{\text {eq }}$ \\
\hline Ni1 & $0.74296(4)$ & $0.67056(3)$ & $0.52192(3)$ & $0.02311(16)$ \\
\hline Fe1 & $0.56172(4)$ & 0.71009 & 0.49879 & $0.02225(17)$ \\
\hline $\mathrm{S} 1$ & $0.68117(7)$ & $0.70012(6)$ & $0.42497(5)$ & $0.0256(3)$ \\
\hline $\mathrm{S} 2$ & $0.79921(7)$ & $0.57496(6)$ & $0.47542(5)$ & 0.0281 \\
\hline $\mathrm{S} 3$ & $0.79344(7)$ & $0.64527(6)$ & $0.62343(5)$ & 0.0268 \\
\hline S4 & $0.67593(7)$ & $0.76476(6)$ & $0.56176(5)$ & 0.0256 \\
\hline $\mathrm{O} 1$ & $0.5610(2)$ & $0.55947(17)$ & $0.54595(17)$ & $0.0398(8)$ \\
\hline $\mathrm{C} 1$ & 0.4278 & $0.7053(2)$ & $0.5115(2)$ & $0.0248(9)$ \\
\hline $\mathrm{C} 2$ & $0.4585(3)$ & $0.7723(2)$ & $0.5392(2)$ & $0.0265(10)$ \\
\hline $\mathrm{C} 3$ & $0.4983(3)$ & $0.8115(2)$ & $0.4831(2)$ & $0.0303(10)$ \\
\hline $\mathrm{C} 4$ & $0.4907(3)$ & $0.7698(2)$ & $0.4222(2)$ & $0.0316(10)$ \\
\hline $\mathrm{C} 5$ & $0.4483(3)$ & $0.7029(2)$ & $0.4388(2)$ & $0.0276(10)$ \\
\hline C6 & 0.3751 & $0.6504(2)$ & $0.5513(2)$ & $0.0353(11)$ \\
\hline H6A & 0.313257 & 0.661374 & 0.545916 & $0.053^{*}$ \\
\hline H6B & 0.390791 & 0.652574 & 0.600690 & $0.053^{*}$ \\
\hline $\mathrm{H} 6 \mathrm{C}$ & 0.387078 & 0.601560 & 0.533256 & $0.053 *$ \\
\hline $\mathrm{C} 7$ & $0.4426(3)$ & $0.7979(3)$ & $0.6122(2)$ & $0.0357(11)$ \\
\hline $\mathrm{H} 7 \mathrm{~A}$ & 0.380057 & 0.803763 & 0.619663 & $0.054^{*}$ \\
\hline H7B & 0.471790 & 0.844604 & 0.619491 & $0.054^{*}$ \\
\hline $\mathrm{H} 7 \mathrm{C}$ & 0.465444 & 0.761895 & 0.645062 & $0.054^{*}$ \\
\hline $\mathrm{C} 8$ & 0.5361 & $0.8871(2)$ & 0.4870 & $0.0452(13)$ \\
\hline H8A & 0.492333 & 0.922612 & 0.472476 & $0.068^{*}$ \\
\hline H8B & 0.586638 & 0.890341 & 0.456183 & $0.068 *$ \\
\hline $\mathrm{H} 8 \mathrm{C}$ & 0.554009 & 0.897320 & 0.534968 & $0.068 *$ \\
\hline $\mathrm{C} 9$ & $0.5187(4)$ & $0.7927(3)$ & $0.3503(2)$ & $0.0456(13)$ \\
\hline H9A & 0.475279 & 0.825676 & 0.330445 & $0.068 *$ \\
\hline H9B & 0.524302 & 0.749593 & 0.320604 & $0.068 *$ \\
\hline $\mathrm{H} 9 \mathrm{C}$ & 0.574773 & 0.817703 & 0.353096 & $0.068 *$ \\
\hline $\mathrm{C} 10$ & $0.4183(3)$ & $0.6449(3)$ & $0.3899(2)$ & $0.0444(13)$ \\
\hline H10A & 0.355519 & 0.649373 & 0.382812 & $0.067 *$ \\
\hline H10B & 0.431411 & 0.596988 & 0.409703 & $0.067^{*}$ \\
\hline $\mathrm{H} 10 \mathrm{C}$ & 0.448205 & 0.650298 & 0.345239 & $0.067^{*}$ \\
\hline C11 & $0.5660(3)$ & $0.6194(3)$ & $0.5293(2)$ & $0.0278(10)$ \\
\hline $\mathrm{C} 12$ & $0.6913(3)$ & $0.6181(2)$ & $0.3746(2)$ & $0.0286(10)$ \\
\hline $\mathrm{C} 13$ & $0.7413(3)$ & $0.5637(2)$ & $0.3961(2)$ & $0.0304(10)$ \\
\hline $\mathrm{C} 14$ & $0.7606(3)$ & $0.4963(2)$ & $0.5252(2)$ & $0.0278(10)$ \\
\hline
\end{tabular}




$\begin{array}{lllll}\text { H14A } & 0.696377 & 0.496663 & 0.526437 & 0.033^{*} \\ \text { H14B } & 0.779375 & 0.450965 & 0.501772 & 0.033^{*} \\ \text { C15 } & 0.7957(3) & 0.4971(2) & 0.5998(2) & 0.0358(11) \\ \text { H15A } & 0.786109 & 0.448644 & 0.620841 & 0.043^{*} \\ \text { H15B } & 0.859172 & 0.505579 & 0.598102 & 0.043^{*} \\ \text { C16 } & 0.7548(3) & 0.5546(2) & 0.6466(2) & 0.0328(11) \\ \text { H16A } & 0.769533 & 0.544153 & 0.695734 & 0.039^{*} \\ \text { H16B } & 0.690822 & 0.552699 & 0.641829 & 0.039^{*} \\ \text { C17 } & 0.7331(3) & 0.7017(2) & 0.6814(2) & 0.0292(10) \\ \text { C18 } & 0.6833(3) & 0.7532(2) & 0.6539(2) & 0.0267(10) \\ \text { C19 } & 0.6439(3) & 0.6191(3) & 0.3059(2) & 0.0397(12) \\ \text { H19A } & 0.673889 & 0.587331 & 0.272713 & 0.060^{*} \\ \text { H19B } & 0.642695 & 0.668917 & 0.287647 & 0.060^{*} \\ \text { H19C } & 0.584335 & 0.601759 & 0.312578 & 0.060^{*} \\ \text { C20 } & 0.7620(3) & 0.4947(3) & 0.3569(2) & 0.0411(12) \\ \text { H20A } & 0.733297 & 0.495786 & 0.311395 & 0.062^{*} \\ \text { H20B } & 0.741350 & 0.452654 & 0.383458 & 0.062^{*} \\ \text { H20C } & 0.824947 & 0.490988 & 0.350310 & 0.062^{*} \\ \text { C21 } & 0.7475(3) & 0.6871(3) & 0.7574(2) & 0.0378(12) \\ \text { H21A } & 0.737458 & 0.731855 & 0.783949 & 0.057^{*} \\ \text { H21B } & 0.807296 & 0.670606 & 0.764737 & 0.057^{*} \\ \text { H21C } & 0.707132 & 0.649328 & 0.773176 & 0.057^{*} \\ \text { C22 } & 0.6369(3) & 0.8090(3) & 0.6972(2) & 0.0381(11) \\ \text { H22A } & 0.676793 & 0.828725 & 0.732196 & 0.057^{*} \\ \text { H22B } & 0.587285 & 0.786252 & 0.720573 & 0.057^{*} \\ \text { H22C } & 0.616285 & 0.848535 & 0.667210 & 0.057^{*} \\ \text { P1 } & 1.000000 & 0.50751(9) & 0.750000 & 0.0338(4) \\ \text { P2 } & 0.500000 & 0.56829(10) & 0.750000 & 0.0361(4) \\ \text { F1 } & 0.9262(3) & 0.44789(18) & 0.7508(2) & 0.0895(14) \\ \text { F2 } & 0.92696(19) & 0.56827(15) & 0.75048(16) & 0.0518(8) \\ \text { F3 } & 0.9995(2) & 0.5074(2) & 0.83285(14) & 0.0700(10) \\ \text { F4 } & 0.4168(2) & 0.5668(2) & 0.7027(2) & 0.0808(11) \\ \text { F5 } & 0.5402(3) & 0.62895(18) & 0.70149(18) & 0.0727(10) \\ \text { F6 } & 0.5418(2) & 0.50624(17) & 0.70251(16) & 0.0670(10) \\ & & & & \end{array}$

Atomic displacement parameters $\left(\AA^{2}\right)$

\begin{tabular}{lllllll}
\hline & $U^{11}$ & $U^{22}$ & $U^{33}$ & $U^{12}$ & $U^{13}$ & $U^{23}$ \\
\hline Ni1 & $0.0223(3)$ & $0.0268(3)$ & $0.0202(3)$ & $-0.0012(2)$ & $-0.0002(2)$ & $-0.0010(2)$ \\
Fe1 & $0.0216(4)$ & $0.0250(3)$ & $0.0201(3)$ & $0.0001(3)$ & $0.0002(2)$ & $0.0023(2)$ \\
S1 & $0.0247(6)$ & $0.0316(6)$ & $0.0204(5)$ & $-0.0003(5)$ & $0.0005(4)$ & $0.0000(4)$ \\
S2 & $0.0229(6)$ & $0.0327(6)$ & $0.0286(6)$ & $0.0013(5)$ & $0.0008(4)$ & $-0.0049(4)$ \\
S3 & $0.0261(6)$ & $0.0303(6)$ & $0.0242(5)$ & $-0.0011(5)$ & $-0.0038(4)$ & $-0.0004(4)$ \\
S4 & $0.0269(6)$ & $0.0269(5)$ & $0.0231(5)$ & $-0.0013(5)$ & $-0.0007(4)$ & $-0.0010(4)$ \\
O1 & $0.036(2)$ & $0.0321(18)$ & $0.051(2)$ & $-0.0015(15)$ & $0.0016(16)$ & $0.0102(15)$ \\
C1 & $0.021(2)$ & $0.028(2)$ & $0.025(2)$ & $0.0042(18)$ & $-0.0034(17)$ & $0.0000(17)$ \\
C2 & $0.022(2)$ & $0.030(2)$ & $0.027(2)$ & $0.0050(18)$ & $-0.0023(18)$ & $0.0014(18)$ \\
C3 & $0.018(2)$ & $0.029(2)$ & $0.044(3)$ & $0.0021(19)$ & $0.001(2)$ & $0.004(2)$
\end{tabular}




$\begin{array}{lllllll}\text { C4 } & 0.025(3) & 0.043(3) & 0.026(2) & 0.009(2) & 0.0030(19) & 0.011(2) \\ \text { C5 } & 0.020(2) & 0.039(3) & 0.024(2) & 0.006(2) & -0.0028(17) & -0.0011(18) \\ \text { C6 } & 0.027(3) & 0.034(2) & 0.046(3) & -0.007(2) & 0.003(2) & 0.003(2) \\ \text { C7 } & 0.031(3) & 0.045(3) & 0.030(2) & 0.005(2) & 0.000(2) & -0.010(2) \\ \text { C8 } & 0.028(3) & 0.028(2) & 0.079(4) & -0.006(2) & 0.007(3) & 0.010(2) \\ \text { C9 } & 0.041(3) & 0.060(3) & 0.036(3) & 0.014(3) & 0.009(2) & 0.021(2) \\ \text { C10 } & 0.036(3) & 0.057(3) & 0.040(3) & 0.007(3) & -0.013(2) & -0.009(2) \\ \text { C11 } & 0.017(2) & 0.039(3) & 0.027(2) & 0.001(2) & 0.0023(17) & 0.001(2) \\ \text { C12 } & 0.027(3) & 0.033(2) & 0.025(2) & -0.002(2) & 0.0040(18) & -0.0060(18) \\ \text { C13 } & 0.029(3) & 0.039(3) & 0.024(2) & 0.001(2) & 0.0034(19) & -0.0076(19) \\ \text { C14 } & 0.023(2) & 0.023(2) & 0.037(2) & 0.0006(18) & -0.0029(19) & -0.0038(18) \\ \text { C15 } & 0.034(3) & 0.032(2) & 0.042(3) & 0.001(2) & -0.006(2) & 0.004(2) \\ \text { C16 } & 0.037(3) & 0.031(2) & 0.031(2) & 0.000(2) & -0.003(2) & 0.0036(19) \\ \text { C17 } & 0.029(3) & 0.035(2) & 0.023(2) & -0.008(2) & -0.0010(19) & -0.0039(19) \\ \text { C18 } & 0.028(3) & 0.030(2) & 0.021(2) & -0.007(2) & -0.0012(18) & -0.0052(17) \\ \text { C19 } & 0.041(3) & 0.052(3) & 0.026(2) & -0.009(3) & -0.002(2) & -0.006(2) \\ \text { C20 } & 0.046(3) & 0.045(3) & 0.033(2) & -0.001(2) & 0.003(2) & -0.014(2) \\ \text { C21 } & 0.044(3) & 0.045(3) & 0.025(2) & -0.003(2) & -0.005(2) & 0.002(2) \\ \text { C22 } & 0.039(3) & 0.048(3) & 0.027(2) & 0.003(2) & -0.005(2) & -0.010(2) \\ \text { P1 } & 0.0388(11) & 0.0305(9) & 0.0321(9) & 0.000 & -0.0043(7) & 0.000 \\ \text { P2 } & 0.0311(10) & 0.0423(10) & 0.0350(9) & 0.000 & -0.0006(8) & 0.000 \\ \text { F1 } & 0.112(4) & 0.055(2) & 0.102(3) & -0.046(2) & 0.036(3) & -0.027(2) \\ \text { F2 } & 0.0310(18) & 0.0563(18) & 0.068(2) & 0.0122(14) & 0.0023(14) & 0.0122(15) \\ \text { F3 } & 0.060(2) & 0.115(3) & 0.0349(16) & 0.010(2) & -0.0033(15) & 0.0145(17) \\ \text { F4 } & 0.061(2) & 0.090(3) & 0.092(3) & -0.011(2) & -0.039(2) & 0.017(2) \\ \text { F5 } & 0.090(3) & 0.0526(19) & 0.075(2) & -0.0221(19) & 0.016(2) & 0.0109(17) \\ \text { F6 } & 0.089(3) & 0.061(2) & 0.0507(18) & 0.0087(19) & 0.0156(18) & -0.0130(15) \\ & & & & & \end{array}$

Geometric parameters $\left(\AA,{ }^{\circ}\right)$

\begin{tabular}{llll}
\hline Ni1-S3 & $2.1507(11)$ & $\mathrm{C} 10-\mathrm{H} 10 \mathrm{~A}$ & 0.9800 \\
$\mathrm{~N} 1-\mathrm{S} 2$ & $2.1530(12)$ & $\mathrm{C} 10-\mathrm{H} 10 \mathrm{~B}$ & 0.9800 \\
$\mathrm{~N} i 1-\mathrm{S} 4$ & $2.1563(12)$ & $\mathrm{C} 10-\mathrm{H} 10 \mathrm{C}$ & 0.9800 \\
$\mathrm{~N} 1-\mathrm{S} 1$ & $2.1616(11)$ & $\mathrm{C} 12-\mathrm{C} 13$ & $1.328(6)$ \\
$\mathrm{Ni1}-\mathrm{Fe} 1$ & $2.9195(8)$ & $\mathrm{C} 12-\mathrm{C} 19$ & $1.510(6)$ \\
$\mathrm{Fe} 1-\mathrm{C} 11$ & $1.768(5)$ & $\mathrm{C} 13-\mathrm{C} 20$ & $1.509(6)$ \\
$\mathrm{Fe} 1-\mathrm{C} 1$ & $2.080(4)$ & $\mathrm{C} 14-\mathrm{C} 15$ & $1.532(6)$ \\
$\mathrm{Fe} 1-\mathrm{C} 5$ & $2.098(4)$ & $\mathrm{C} 14-\mathrm{H} 14 \mathrm{~A}$ & 0.9900 \\
$\mathrm{Fe} 1-\mathrm{C} 2$ & $2.107(4)$ & $\mathrm{C} 14-\mathrm{H} 14 \mathrm{~B}$ & 0.9900 \\
$\mathrm{Fe} 1-\mathrm{C} 3$ & $2.126(4)$ & $\mathrm{C} 15-\mathrm{C} 16$ & $1.524(6)$ \\
$\mathrm{Fe} 1-\mathrm{C} 4$ & $2.138(4)$ & $\mathrm{C} 15-\mathrm{H} 15 \mathrm{~A}$ & 0.9900 \\
$\mathrm{Fe} 1-\mathrm{S} 1$ & $2.3309(12)$ & $\mathrm{C} 15-\mathrm{H} 15 \mathrm{~B}$ & 0.9900 \\
$\mathrm{Fe} 1-\mathrm{S} 4$ & $2.3602(12)$ & $\mathrm{C} 16-\mathrm{H} 16 \mathrm{~A}$ & 0.9900 \\
$\mathrm{~S} 1-\mathrm{C} 12$ & $1.798(4)$ & $\mathrm{C} 16-\mathrm{H} 16 \mathrm{~B}$ & 0.9900 \\
$\mathrm{~S} 2-\mathrm{C} 13$ & $1.778(4)$ & $\mathrm{C} 17-\mathrm{C} 18$ & $1.329(6)$ \\
$\mathrm{S} 2-\mathrm{C} 14$ & $1.833(4)$ & $\mathrm{C} 17-\mathrm{C} 21$ & $1.501(6)$ \\
$\mathrm{S} 3-\mathrm{C} 17$ & $1.783(5)$ & $\mathrm{C} 18-\mathrm{C} 22$ & $1.503(6)$ \\
$\mathrm{S} 3-\mathrm{C} 16$ & $1.825(4)$ & $\mathrm{C} 19-\mathrm{H} 19 \mathrm{~A}$ & 0.9800
\end{tabular}




\begin{tabular}{|c|c|c|c|}
\hline $\mathrm{S} 4-\mathrm{C} 18$ & $1.786(4)$ & C19-H19B & 0.9800 \\
\hline $\mathrm{O} 1-\mathrm{C} 11$ & $1.149(5)$ & $\mathrm{C} 19-\mathrm{H} 19 \mathrm{C}$ & 0.9800 \\
\hline $\mathrm{C} 1-\mathrm{C} 2$ & $1.423(6)$ & $\mathrm{C} 20-\mathrm{H} 20 \mathrm{~A}$ & 0.9800 \\
\hline $\mathrm{C} 1-\mathrm{C} 5$ & $1.432(6)$ & $\mathrm{C} 20-\mathrm{H} 20 \mathrm{~B}$ & 0.9800 \\
\hline $\mathrm{C} 1-\mathrm{C} 6$ & $1.504(6)$ & $\mathrm{C} 20-\mathrm{H} 20 \mathrm{C}$ & 0.9800 \\
\hline $\mathrm{C} 2-\mathrm{C} 3$ & $1.434(6)$ & $\mathrm{C} 21-\mathrm{H} 21 \mathrm{~A}$ & 0.9800 \\
\hline $\mathrm{C} 2-\mathrm{C} 7$ & $1.499(6)$ & $\mathrm{C} 21-\mathrm{H} 21 \mathrm{~B}$ & 0.9800 \\
\hline $\mathrm{C} 3-\mathrm{C} 4$ & $1.404(6)$ & $\mathrm{C} 21-\mathrm{H} 21 \mathrm{C}$ & 0.9800 \\
\hline $\mathrm{C} 3-\mathrm{C} 8$ & $1.508(6)$ & $\mathrm{C} 22-\mathrm{H} 22 \mathrm{~A}$ & 0.9800 \\
\hline $\mathrm{C} 4-\mathrm{C} 5$ & $1.429(6)$ & $\mathrm{C} 22-\mathrm{H} 22 \mathrm{~B}$ & 0.9800 \\
\hline $\mathrm{C} 4-\mathrm{C} 9$ & $1.507(6)$ & $\mathrm{C} 22-\mathrm{H} 22 \mathrm{C}$ & 0.9800 \\
\hline $\mathrm{C} 5-\mathrm{C} 10$ & $1.493(6)$ & $\mathrm{P} 1-\mathrm{F} 1^{\mathrm{i}}$ & 1.579 \\
\hline C6-H6A & 0.9800 & $\mathrm{P} 1-\mathrm{F} 1$ & $1.579(3)$ \\
\hline C6-H6B & 0.9800 & $\mathrm{P} 1-\mathrm{F} 2$ & $1.585(3)$ \\
\hline C6- $\mathrm{H} 6 \mathrm{C}$ & 0.9800 & $\mathrm{P} 1-\mathrm{F} 2^{\mathrm{i}}$ & $1.585(3)$ \\
\hline C7-H7A & 0.9800 & $\mathrm{P} 1-\mathrm{F} 3$ & $1.592(3)$ \\
\hline C7-H7B & 0.9800 & $\mathrm{P} 1-\mathrm{F} 3^{\mathrm{i}}$ & $1.592(3)$ \\
\hline $\mathrm{C} 7-\mathrm{H} 7 \mathrm{C}$ & 0.9800 & $\mathrm{P} 2-\mathrm{F} 4^{\mathrm{ii}}$ & $1.572(3)$ \\
\hline $\mathrm{C} 8-\mathrm{H} 8 \mathrm{~A}$ & 0.9800 & $\mathrm{P} 2-\mathrm{F} 4$ & $1.572(3)$ \\
\hline $\mathrm{C} 8-\mathrm{H} 8 \mathrm{~B}$ & 0.9800 & $\mathrm{P} 2-\mathrm{F}^{\mathrm{ii}}$ & $1.580(3)$ \\
\hline $\mathrm{C} 8-\mathrm{H} 8 \mathrm{C}$ & 0.9800 & $\mathrm{P} 2-\mathrm{F} 5$ & $1.580(3)$ \\
\hline C9-H9A & 0.9800 & $\mathrm{P} 2-\mathrm{F} 6^{\mathrm{ii}}$ & $1.596(3)$ \\
\hline C9-H9B & 0.9800 & $\mathrm{P} 2-\mathrm{F} 6$ & $1.596(3)$ \\
\hline C9-H9C & 0.9800 & & \\
\hline $\mathrm{S} 3-\mathrm{Ni1}-\mathrm{S} 2$ & $93.13(4)$ & $\mathrm{H} 8 \mathrm{~A}-\mathrm{C} 8-\mathrm{H} 8 \mathrm{~B}$ & 109.5 \\
\hline $\mathrm{S} 3-\mathrm{Ni1}-\mathrm{S} 4$ & $91.41(4)$ & $\mathrm{C} 3-\mathrm{C} 8-\mathrm{H} 8 \mathrm{C}$ & 109.5 \\
\hline $\mathrm{S} 2-\mathrm{Ni1}-\mathrm{S} 4$ & $174.38(5)$ & $\mathrm{H} 8 \mathrm{~A}-\mathrm{C} 8-\mathrm{H} 8 \mathrm{C}$ & 109.5 \\
\hline $\mathrm{S} 3-\mathrm{Ni1}-\mathrm{S} 1$ & $174.42(5)$ & $\mathrm{H} 8 \mathrm{~B}-\mathrm{C} 8-\mathrm{H} 8 \mathrm{C}$ & 109.5 \\
\hline $\mathrm{S} 2-\mathrm{Ni1}-\mathrm{S} 1$ & $91.42(4)$ & $\mathrm{C} 4-\mathrm{C} 9-\mathrm{H} 9 \mathrm{~A}$ & 109.5 \\
\hline $\mathrm{S} 4-\mathrm{Ni} 1-\mathrm{S} 1$ & $83.87(4)$ & $\mathrm{C} 4-\mathrm{C} 9-\mathrm{H} 9 \mathrm{~B}$ & 109.5 \\
\hline $\mathrm{S} 3-\mathrm{Ni1}-\mathrm{Fe} 1$ & $122.53(4)$ & $\mathrm{H} 9 \mathrm{~A}-\mathrm{C} 9-\mathrm{H} 9 \mathrm{~B}$ & 109.5 \\
\hline $\mathrm{S} 2-\mathrm{Ni} 1-\mathrm{Fe} 1$ & $121.66(4)$ & $\mathrm{C} 4-\mathrm{C} 9-\mathrm{H} 9 \mathrm{C}$ & 109.5 \\
\hline $\mathrm{S} 4-\mathrm{Ni} 1-\mathrm{Fe} 1$ & $52.85(3)$ & $\mathrm{H} 9 \mathrm{~A}-\mathrm{C} 9-\mathrm{H} 9 \mathrm{C}$ & 109.5 \\
\hline $\mathrm{S} 1-\mathrm{Ni1}-\mathrm{Fe} 1$ & $52.04(3)$ & $\mathrm{H} 9 \mathrm{~B}-\mathrm{C} 9-\mathrm{H} 9 \mathrm{C}$ & 109.5 \\
\hline $\mathrm{C} 11-\mathrm{Fe} 1-\mathrm{C} 1$ & $87.63(18)$ & $\mathrm{C} 5-\mathrm{C} 10-\mathrm{H} 10 \mathrm{~A}$ & 109.5 \\
\hline $\mathrm{C} 11-\mathrm{Fe} 1-\mathrm{C} 5$ & $98.85(18)$ & $\mathrm{C} 5-\mathrm{C} 10-\mathrm{H} 10 \mathrm{~B}$ & 109.5 \\
\hline $\mathrm{C} 1-\mathrm{Fe} 1-\mathrm{C} 5$ & $40.08(15)$ & $\mathrm{H} 10 \mathrm{~A}-\mathrm{C} 10-\mathrm{H} 10 \mathrm{~B}$ & 109.5 \\
\hline $\mathrm{C} 11-\mathrm{Fe} 1-\mathrm{C} 2$ & $114.69(18)$ & $\mathrm{C} 5-\mathrm{C} 10-\mathrm{H} 10 \mathrm{C}$ & 109.5 \\
\hline $\mathrm{C} 1-\mathrm{Fe} 1-\mathrm{C} 2$ & $39.73(15)$ & $\mathrm{H} 10 \mathrm{~A}-\mathrm{C} 10-\mathrm{H} 10 \mathrm{C}$ & 109.5 \\
\hline $\mathrm{C} 5-\mathrm{Fe} 1-\mathrm{C} 2$ & $66.92(16)$ & $\mathrm{H} 10 \mathrm{~B}-\mathrm{C} 10-\mathrm{H} 10 \mathrm{C}$ & 109.5 \\
\hline $\mathrm{C} 11-\mathrm{Fe} 1-\mathrm{C} 3$ & $152.97(19)$ & $\mathrm{O} 1-\mathrm{C} 11-\mathrm{Fe} 1$ & $173.2(4)$ \\
\hline $\mathrm{C} 1-\mathrm{Fe} 1-\mathrm{C} 3$ & $66.26(16)$ & $\mathrm{C} 13-\mathrm{C} 12-\mathrm{C} 19$ & $124.2(4)$ \\
\hline $\mathrm{C} 5-\mathrm{Fe} 1-\mathrm{C} 3$ & $66.07(17)$ & $\mathrm{C} 13-\mathrm{C} 12-\mathrm{S} 1$ & $120.9(3)$ \\
\hline $\mathrm{C} 2-\mathrm{Fe} 1-\mathrm{C} 3$ & $39.61(16)$ & $\mathrm{C} 19-\mathrm{C} 12-\mathrm{S} 1$ & $114.7(3)$ \\
\hline $\mathrm{C} 11-\mathrm{Fe} 1-\mathrm{C} 4$ & $137.02(19)$ & $\mathrm{C} 12-\mathrm{C} 13-\mathrm{C} 20$ & $126.9(4)$ \\
\hline $\mathrm{C} 1-\mathrm{Fe} 1-\mathrm{C} 4$ & $66.05(16)$ & $\mathrm{C} 12-\mathrm{C} 13-\mathrm{S} 2$ & $118.0(3)$ \\
\hline $\mathrm{C} 5-\mathrm{Fe} 1-\mathrm{C} 4$ & $39.42(17)$ & $\mathrm{C} 20-\mathrm{C} 13-\mathrm{S} 2$ & $114.8(3)$ \\
\hline
\end{tabular}




\begin{tabular}{|c|c|c|c|}
\hline $\mathrm{C} 2-\mathrm{Fe} 1-\mathrm{C} 4$ & $65.71(16)$ & $\mathrm{C} 15-\mathrm{C} 14-\mathrm{S} 2$ & $111.4(3)$ \\
\hline $\mathrm{C} 3-\mathrm{Fe} 1-\mathrm{C} 4$ & $38.43(17)$ & $\mathrm{C} 15-\mathrm{C} 14-\mathrm{H} 14 \mathrm{~A}$ & 109.3 \\
\hline $\mathrm{C} 11-\mathrm{Fe} 1-\mathrm{S} 1$ & $95.64(14)$ & $\mathrm{S} 2-\mathrm{C} 14-\mathrm{H} 14 \mathrm{~A}$ & 109.3 \\
\hline $\mathrm{C} 1-\mathrm{Fe} 1-\mathrm{S} 1$ & $148.36(12)$ & $\mathrm{C} 15-\mathrm{C} 14-\mathrm{H} 14 \mathrm{~B}$ & 109.3 \\
\hline $\mathrm{C} 5-\mathrm{Fe} 1-\mathrm{S} 1$ & $108.58(12)$ & $\mathrm{S} 2-\mathrm{C} 14-\mathrm{H} 14 \mathrm{~B}$ & 109.3 \\
\hline $\mathrm{C} 2-\mathrm{Fe} 1-\mathrm{S} 1$ & $149.63(12)$ & $\mathrm{H} 14 \mathrm{~A}-\mathrm{C} 14-\mathrm{H} 14 \mathrm{~B}$ & 108.0 \\
\hline $\mathrm{C} 3-\mathrm{Fe} 1-\mathrm{S} 1$ & $110.20(12)$ & $\mathrm{C} 16-\mathrm{C} 15-\mathrm{C} 14$ & $114.4(4)$ \\
\hline $\mathrm{C} 4-\mathrm{Fe} 1-\mathrm{S} 1$ & $91.47(12)$ & $\mathrm{C} 16-\mathrm{C} 15-\mathrm{H} 15 \mathrm{~A}$ & 108.7 \\
\hline $\mathrm{C} 11-\mathrm{Fe} 1-\mathrm{S} 4$ & $101.72(14)$ & $\mathrm{C} 14-\mathrm{C} 15-\mathrm{H} 15 \mathrm{~A}$ & 108.7 \\
\hline $\mathrm{C} 1-\mathrm{Fe} 1-\mathrm{S} 4$ & $134.25(11)$ & $\mathrm{C} 16-\mathrm{C} 15-\mathrm{H} 15 \mathrm{~B}$ & 108.7 \\
\hline $\mathrm{C} 5-\mathrm{Fe} 1-\mathrm{S} 4$ & $158.40(12)$ & $\mathrm{C} 14-\mathrm{C} 15-\mathrm{H} 15 \mathrm{~B}$ & 108.7 \\
\hline $\mathrm{C} 2-\mathrm{Fe} 1-\mathrm{S} 4$ & $98.20(12)$ & $\mathrm{H} 15 \mathrm{~A}-\mathrm{C} 15-\mathrm{H} 15 \mathrm{~B}$ & 107.6 \\
\hline $\mathrm{C} 3-\mathrm{Fe} 1-\mathrm{S} 4$ & $92.43(13)$ & $\mathrm{C} 15-\mathrm{C} 16-\mathrm{S} 3$ & $110.7(3)$ \\
\hline $\mathrm{C} 4-\mathrm{Fe} 1-\mathrm{S} 4$ & $121.08(13)$ & $\mathrm{C} 15-\mathrm{C} 16-\mathrm{H} 16 \mathrm{~A}$ & 109.5 \\
\hline $\mathrm{S} 1-\mathrm{Fe} 1-\mathrm{S} 4$ & $75.92(4)$ & $\mathrm{S} 3-\mathrm{C} 16-\mathrm{H} 16 \mathrm{~A}$ & 109.5 \\
\hline $\mathrm{C} 11-\mathrm{Fe} 1-\mathrm{Ni} 1$ & $71.28(14)$ & $\mathrm{C} 15-\mathrm{C} 16-\mathrm{H} 16 \mathrm{~B}$ & 109.5 \\
\hline $\mathrm{C} 1-\mathrm{Fe} 1-\mathrm{Ni} 1$ & $157.04(11)$ & $\mathrm{S} 3-\mathrm{C} 16-\mathrm{H} 16 \mathrm{~B}$ & 109.5 \\
\hline $\mathrm{C} 5-\mathrm{Fe} 1-\mathrm{Ni} 1$ & $149.87(12)$ & $\mathrm{H} 16 \mathrm{~A}-\mathrm{C} 16-\mathrm{H} 16 \mathrm{~B}$ & 108.1 \\
\hline $\mathrm{C} 2-\mathrm{Fe} 1-\mathrm{Ni} 1$ & $143.21(12)$ & $\mathrm{C} 18-\mathrm{C} 17-\mathrm{C} 21$ & $126.8(4)$ \\
\hline $\mathrm{C} 3-\mathrm{Fe} 1-\mathrm{Ni} 1$ & $132.80(12)$ & $\mathrm{C} 18-\mathrm{C} 17-\mathrm{S} 3$ & $117.8(3)$ \\
\hline $\mathrm{C} 4-\mathrm{Fe} 1-\mathrm{Ni} 1$ & $136.25(12)$ & $\mathrm{C} 21-\mathrm{C} 17-\mathrm{S} 3$ & $115.3(3)$ \\
\hline $\mathrm{S} 1-\mathrm{Fe} 1-\mathrm{Ni} 1$ & $46.99(3)$ & $\mathrm{C} 17-\mathrm{C} 18-\mathrm{C} 22$ & $122.7(4)$ \\
\hline $\mathrm{S} 4-\mathrm{Fe} 1-\mathrm{Ni} 1$ & $46.74(3)$ & $\mathrm{C} 17-\mathrm{C} 18-\mathrm{S} 4$ & $121.1(3)$ \\
\hline $\mathrm{C} 12-\mathrm{S} 1-\mathrm{Ni1}$ & $102.40(15)$ & $\mathrm{C} 22-\mathrm{C} 18-\mathrm{S} 4$ & $116.0(3)$ \\
\hline $\mathrm{C} 12-\mathrm{S} 1-\mathrm{Fe} 1$ & $117.49(15)$ & $\mathrm{C} 12-\mathrm{C} 19-\mathrm{H} 19 \mathrm{~A}$ & 109.5 \\
\hline $\mathrm{Ni} 1-\mathrm{S} 1-\mathrm{Fe} 1$ & $80.97(4)$ & $\mathrm{C} 12-\mathrm{C} 19-\mathrm{H} 19 \mathrm{~B}$ & 109.5 \\
\hline $\mathrm{C} 13-\mathrm{S} 2-\mathrm{C} 14$ & $101.1(2)$ & $\mathrm{H} 19 \mathrm{~A}-\mathrm{C} 19-\mathrm{H} 19 \mathrm{~B}$ & 109.5 \\
\hline $\mathrm{C} 13-\mathrm{S} 2-\mathrm{Ni1}$ & $104.38(15)$ & $\mathrm{C} 12-\mathrm{C} 19-\mathrm{H} 19 \mathrm{C}$ & 109.5 \\
\hline $\mathrm{C} 14-\mathrm{S} 2-\mathrm{Ni1}$ & $107.23(14)$ & $\mathrm{H} 19 \mathrm{~A}-\mathrm{C} 19-\mathrm{H} 19 \mathrm{C}$ & 109.5 \\
\hline $\mathrm{C} 17-\mathrm{S} 3-\mathrm{C} 16$ & $102.0(2)$ & $\mathrm{H} 19 \mathrm{~B}-\mathrm{C} 19-\mathrm{H} 19 \mathrm{C}$ & 109.5 \\
\hline $\mathrm{C} 17-\mathrm{S} 3-\mathrm{Ni} 1$ & $104.64(15)$ & $\mathrm{C} 13-\mathrm{C} 20-\mathrm{H} 20 \mathrm{~A}$ & 109.5 \\
\hline $\mathrm{C} 16-\mathrm{S} 3-\mathrm{Ni} 1$ & $107.48(15)$ & $\mathrm{C} 13-\mathrm{C} 20-\mathrm{H} 20 \mathrm{~B}$ & 109.5 \\
\hline $\mathrm{C} 18-\mathrm{S} 4-\mathrm{Ni} 1$ & $103.04(15)$ & $\mathrm{H} 20 \mathrm{~A}-\mathrm{C} 20-\mathrm{H} 20 \mathrm{~B}$ & 109.5 \\
\hline $\mathrm{C} 18-\mathrm{S} 4-\mathrm{Fe} 1$ & $120.33(15)$ & $\mathrm{C} 13-\mathrm{C} 20-\mathrm{H} 20 \mathrm{C}$ & 109.5 \\
\hline $\mathrm{Ni} 1-\mathrm{S} 4-\mathrm{Fe} 1$ & $80.41(4)$ & $\mathrm{H} 20 \mathrm{~A}-\mathrm{C} 20-\mathrm{H} 20 \mathrm{C}$ & 109.5 \\
\hline $\mathrm{C} 2-\mathrm{C} 1-\mathrm{C} 5$ & $108.6(4)$ & $\mathrm{H} 20 \mathrm{~B}-\mathrm{C} 20-\mathrm{H} 20 \mathrm{C}$ & 109.5 \\
\hline $\mathrm{C} 2-\mathrm{C} 1-\mathrm{C} 6$ & $124.7(4)$ & $\mathrm{C} 17-\mathrm{C} 21-\mathrm{H} 21 \mathrm{~A}$ & 109.5 \\
\hline $\mathrm{C} 5-\mathrm{C} 1-\mathrm{C} 6$ & $126.4(4)$ & $\mathrm{C} 17-\mathrm{C} 21-\mathrm{H} 21 \mathrm{~B}$ & 109.5 \\
\hline $\mathrm{C} 2-\mathrm{C} 1-\mathrm{Fe} 1$ & $71.2(2)$ & $\mathrm{H} 21 \mathrm{~A}-\mathrm{C} 21-\mathrm{H} 21 \mathrm{~B}$ & 109.5 \\
\hline $\mathrm{C} 5-\mathrm{C} 1-\mathrm{Fe} 1$ & $70.6(2)$ & $\mathrm{C} 17-\mathrm{C} 21-\mathrm{H} 21 \mathrm{C}$ & 109.5 \\
\hline $\mathrm{C} 6-\mathrm{C} 1-\mathrm{Fe} 1$ & $128.5(3)$ & $\mathrm{H} 21 \mathrm{~A}-\mathrm{C} 21-\mathrm{H} 21 \mathrm{C}$ & 109.5 \\
\hline $\mathrm{C} 1-\mathrm{C} 2-\mathrm{C} 3$ & $107.2(4)$ & $\mathrm{H} 21 \mathrm{~B}-\mathrm{C} 21-\mathrm{H} 21 \mathrm{C}$ & 109.5 \\
\hline $\mathrm{C} 1-\mathrm{C} 2-\mathrm{C} 7$ & $124.5(4)$ & $\mathrm{C} 18-\mathrm{C} 22-\mathrm{H} 22 \mathrm{~A}$ & 109.5 \\
\hline $\mathrm{C} 3-\mathrm{C} 2-\mathrm{C} 7$ & $128.1(4)$ & $\mathrm{C} 18-\mathrm{C} 22-\mathrm{H} 22 \mathrm{~B}$ & 109.5 \\
\hline $\mathrm{C} 1-\mathrm{C} 2-\mathrm{Fe} 1$ & $69.1(2)$ & $\mathrm{H} 22 \mathrm{~A}-\mathrm{C} 22-\mathrm{H} 22 \mathrm{~B}$ & 109.5 \\
\hline $\mathrm{C} 3-\mathrm{C} 2-\mathrm{Fe} 1$ & $70.9(2)$ & $\mathrm{C} 18-\mathrm{C} 22-\mathrm{H} 22 \mathrm{C}$ & 109.5 \\
\hline $\mathrm{C} 7-\mathrm{C} 2-\mathrm{Fe} 1$ & $129.7(3)$ & $\mathrm{H} 22 \mathrm{~A}-\mathrm{C} 22-\mathrm{H} 22 \mathrm{C}$ & 109.5 \\
\hline $\mathrm{C} 4-\mathrm{C} 3-\mathrm{C} 2$ & $108.5(4)$ & $\mathrm{H} 22 \mathrm{~B}-\mathrm{C} 22-\mathrm{H} 22 \mathrm{C}$ & 109.5 \\
\hline
\end{tabular}




\begin{tabular}{|c|c|c|c|}
\hline $\mathrm{C} 4-\mathrm{C} 3-\mathrm{C} 8$ & $125.2(4)$ & $\mathrm{F} 1-\mathrm{i} 1-\mathrm{F} 1$ & $92.2(3)$ \\
\hline $\mathrm{C} 2-\mathrm{C} 3-\mathrm{C} 8$ & $126.1(4)$ & $\mathrm{F} 1{ }^{\mathrm{i}}-\mathrm{P} 1-\mathrm{F} 2$ & $179.1(2)$ \\
\hline $\mathrm{C} 4-\mathrm{C} 3-\mathrm{Fe} 1$ & $71.3(2)$ & $\mathrm{F} 1-\mathrm{P} 1-\mathrm{F} 2$ & $88.70(19)$ \\
\hline $\mathrm{C} 2-\mathrm{C} 3-\mathrm{Fe} 1$ & $69.5(2)$ & $\mathrm{F} 1^{\mathrm{i}}-\mathrm{P} 1-\mathrm{F} 2^{\mathrm{i}}$ & $88.70(19)$ \\
\hline $\mathrm{C} 8-\mathrm{C} 3-\mathrm{Fe} 1$ & $128.5(3)$ & $\mathrm{F} 1-\mathrm{P} 1-\mathrm{F} 2^{\mathrm{i}}$ & $179.11(19)$ \\
\hline $\mathrm{C} 3-\mathrm{C} 4-\mathrm{C} 5$ & $108.8(4)$ & $\mathrm{F} 2-\mathrm{P} 1-\mathrm{F} 2^{\mathrm{i}}$ & $90.4(2)$ \\
\hline $\mathrm{C} 3-\mathrm{C} 4-\mathrm{C} 9$ & $126.0(4)$ & $\mathrm{F} 1{ }^{\mathrm{i}}-\mathrm{P} 1-\mathrm{F} 3$ & $90.73(19)$ \\
\hline $\mathrm{C} 5-\mathrm{C} 4-\mathrm{C} 9$ & $125.2(4)$ & $\mathrm{F} 1-\mathrm{P} 1-\mathrm{F} 3$ & $89.21(19)$ \\
\hline $\mathrm{C} 3-\mathrm{C} 4-\mathrm{Fe} 1$ & $70.3(2)$ & $\mathrm{F} 2-\mathrm{P} 1-\mathrm{F} 3$ & $89.51(17)$ \\
\hline $\mathrm{C} 5-\mathrm{C} 4-\mathrm{Fe} 1$ & $68.8(2)$ & $\mathrm{F} 2 \mathrm{i}-\mathrm{P} 1-\mathrm{F} 3$ & $90.56(17)$ \\
\hline $\mathrm{C} 9-\mathrm{C} 4-\mathrm{Fe} 1$ & $128.9(3)$ & $\mathrm{F} 1^{\mathrm{i}}-\mathrm{P} 1-\mathrm{F} 3^{\mathrm{i}}$ & $89.21(19)$ \\
\hline $\mathrm{C} 4-\mathrm{C} 5-\mathrm{C} 1$ & $107.0(4)$ & $\mathrm{F} 1-\mathrm{P} 1-\mathrm{F} 3^{\mathrm{i}}$ & $90.73(19)$ \\
\hline $\mathrm{C} 4-\mathrm{C} 5-\mathrm{C} 10$ & $127.9(4)$ & $\mathrm{F} 2-\mathrm{P} 1-\mathrm{F} 3^{\mathrm{i}}$ & $90.56(17)$ \\
\hline $\mathrm{C} 1-\mathrm{C} 5-\mathrm{C} 10$ & $124.6(4)$ & $\mathrm{F} 2^{\mathrm{i}}-\mathrm{P} 1-\mathrm{F} 3^{\mathrm{i}}$ & $89.51(17)$ \\
\hline $\mathrm{C} 4-\mathrm{C} 5-\mathrm{Fe} 1$ & $71.8(2)$ & $\mathrm{F} 3-\mathrm{P} 1-\mathrm{F}^{\mathrm{i}}$ & $179.9(3)$ \\
\hline $\mathrm{C} 1-\mathrm{C} 5-\mathrm{Fe} 1$ & $69.3(2)$ & $\mathrm{F} 4{ }^{\mathrm{ii}}-\mathrm{P} 2-\mathrm{F} 4$ & $178.0(3)$ \\
\hline $\mathrm{C} 10-\mathrm{C} 5-\mathrm{Fe} 1$ & $130.4(3)$ & $\mathrm{F} 4^{\mathrm{ii}}-\mathrm{P} 2-\mathrm{F} 5^{\mathrm{ii}}$ & $89.5(2)$ \\
\hline $\mathrm{C} 1-\mathrm{C} 6-\mathrm{H} 6 \mathrm{~A}$ & 109.5 & $\mathrm{~F} 4-\mathrm{P} 2-\mathrm{F} 5^{\mathrm{ii}}$ & $91.9(2)$ \\
\hline $\mathrm{C} 1-\mathrm{C} 6-\mathrm{H} 6 \mathrm{~B}$ & 109.5 & $\mathrm{~F} 4{ }^{\mathrm{ii}}-\mathrm{P} 2-\mathrm{F} 5$ & $91.9(2)$ \\
\hline $\mathrm{H} 6 \mathrm{~A}-\mathrm{C} 6-\mathrm{H} 6 \mathrm{~B}$ & 109.5 & $\mathrm{~F} 4-\mathrm{P} 2-\mathrm{F} 5$ & $89.5(2)$ \\
\hline $\mathrm{C} 1-\mathrm{C} 6-\mathrm{H} 6 \mathrm{C}$ & 109.5 & 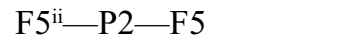 & $90.2(3)$ \\
\hline $\mathrm{H} 6 \mathrm{~A}-\mathrm{C} 6-\mathrm{H} 6 \mathrm{C}$ & 109.5 & $\mathrm{~F} 4^{\mathrm{ii}}-\mathrm{P} 2-\mathrm{F} 6^{\mathrm{ii}}$ & $89.2(2)$ \\
\hline $\mathrm{H} 6 \mathrm{~B}-\mathrm{C} 6-\mathrm{H} 6 \mathrm{C}$ & 109.5 & $\mathrm{~F} 4-\mathrm{P} 2-\mathrm{F} 6^{\mathrm{ii}}$ & $89.4(2)$ \\
\hline $\mathrm{C} 2-\mathrm{C} 7-\mathrm{H} 7 \mathrm{~A}$ & 109.5 & $\mathrm{~F} 5^{\mathrm{ii}}-\mathrm{P} 2-\mathrm{F} 6^{\mathrm{ii}}$ & $90.47(18)$ \\
\hline $\mathrm{C} 2-\mathrm{C} 7-\mathrm{H} 7 \mathrm{~B}$ & 109.5 & $\mathrm{~F} 5-\mathrm{P} 2-\mathrm{F} 6^{\mathrm{ii}}$ & $178.67(19)$ \\
\hline $\mathrm{H} 7 \mathrm{~A}-\mathrm{C} 7-\mathrm{H} 7 \mathrm{~B}$ & 109.5 & $\mathrm{~F} 4{ }^{\mathrm{ii}}-\mathrm{P} 2-\mathrm{F} 6$ & $89.4(2)$ \\
\hline $\mathrm{C} 2-\mathrm{C} 7-\mathrm{H} 7 \mathrm{C}$ & 109.5 & $\mathrm{~F} 4-\mathrm{P} 2-\mathrm{F} 6$ & $89.2(2)$ \\
\hline $\mathrm{H} 7 \mathrm{~A}-\mathrm{C} 7-\mathrm{H} 7 \mathrm{C}$ & 109.5 & $\mathrm{~F} 5{ }^{\mathrm{ii}}-\mathrm{P} 2-\mathrm{F} 6$ & $178.66(19)$ \\
\hline $\mathrm{H} 7 \mathrm{~B}-\mathrm{C} 7-\mathrm{H} 7 \mathrm{C}$ & 109.5 & $\mathrm{~F} 5-\mathrm{P} 2-\mathrm{F} 6$ & $90.47(18)$ \\
\hline $\mathrm{C} 3-\mathrm{C} 8-\mathrm{H} 8 \mathrm{~A}$ & 109.5 & $\mathrm{~F} 6^{\mathrm{ii}}-\mathrm{P} 2-\mathrm{F} 6$ & $88.8(3)$ \\
\hline $\mathrm{C} 3-\mathrm{C} 8-\mathrm{H} 8 \mathrm{~B}$ & 109.5 & & \\
\hline $\mathrm{C} 5-\mathrm{C} 1-\mathrm{C} 2-\mathrm{C} 3$ & $0.0(5)$ & $\mathrm{C} 2-\mathrm{C} 1-\mathrm{C} 5-\mathrm{C} 10$ & $-173.1(4)$ \\
\hline $\mathrm{C} 6-\mathrm{C} 1-\mathrm{C} 2-\mathrm{C} 3$ & $-174.6(4)$ & $\mathrm{C} 6-\mathrm{C} 1-\mathrm{C} 5-\mathrm{C} 10$ & $1.4(7)$ \\
\hline $\mathrm{Fe} 1-\mathrm{C} 1-\mathrm{C} 2-\mathrm{C} 3$ & $61.0(3)$ & $\mathrm{Fe} 1-\mathrm{C} 1-\mathrm{C} 5-\mathrm{C} 10$ & $125.5(4)$ \\
\hline $\mathrm{C} 5-\mathrm{C} 1-\mathrm{C} 2-\mathrm{C} 7$ & $174.4(4)$ & $\mathrm{C} 2-\mathrm{C} 1-\mathrm{C} 5-\mathrm{Fe} 1$ & $61.4(3)$ \\
\hline $\mathrm{C} 6-\mathrm{C} 1-\mathrm{C} 2-\mathrm{C} 7$ & $-0.2(7)$ & $\mathrm{C} 6-\mathrm{C} 1-\mathrm{C} 5-\mathrm{Fe} 1$ & $-124.2(4)$ \\
\hline $\mathrm{Fe} 1-\mathrm{C} 1-\mathrm{C} 2-\mathrm{C} 7$ & $-124.5(4)$ & $\mathrm{Ni} 1-\mathrm{S} 1-\mathrm{C} 12-\mathrm{C} 13$ & $-10.7(4)$ \\
\hline $\mathrm{C} 5-\mathrm{C} 1-\mathrm{C} 2-\mathrm{Fe} 1$ & $-61.0(3)$ & $\mathrm{Fe} 1-\mathrm{S} 1-\mathrm{C} 12-\mathrm{C} 13$ & $-96.8(4)$ \\
\hline $\mathrm{C} 6-\mathrm{C} 1-\mathrm{C} 2-\mathrm{Fe} 1$ & $124.4(4)$ & $\mathrm{Ni1}-\mathrm{S} 1-\mathrm{C} 12-\mathrm{C} 19$ & 173.1 \\
\hline $\mathrm{C} 1-\mathrm{C} 2-\mathrm{C} 3-\mathrm{C} 4$ & $1.0(5)$ & $\mathrm{Fe} 1-\mathrm{S} 1-\mathrm{C} 12-\mathrm{C} 19$ & $86.9(3)$ \\
\hline $\mathrm{C} 7-\mathrm{C} 2-\mathrm{C} 3-\mathrm{C} 4$ & $-173.2(4)$ & $\mathrm{C} 19-\mathrm{C} 12-\mathrm{C} 13-\mathrm{C} 20$ & $1.5(8)$ \\
\hline $\mathrm{Fe} 1-\mathrm{C} 2-\mathrm{C} 3-\mathrm{C} 4$ & $60.9(3)$ & $\mathrm{S} 1-\mathrm{C} 12-\mathrm{C} 13-\mathrm{C} 20$ & $-174.4(4)$ \\
\hline $\mathrm{C} 1-\mathrm{C} 2-\mathrm{C} 3-\mathrm{C} 8$ & $176.8(4)$ & $\mathrm{C} 19-\mathrm{C} 12-\mathrm{C} 13-\mathrm{S} 2$ & $174.9(3)$ \\
\hline $\mathrm{C} 7-\mathrm{C} 2-\mathrm{C} 3-\mathrm{C} 8$ & $2.6(8)$ & $\mathrm{S} 1-\mathrm{C} 12-\mathrm{C} 13-\mathrm{S} 2$ & $-1.0(5)$ \\
\hline $\mathrm{Fe} 1-\mathrm{C} 2-\mathrm{C} 3-\mathrm{C} 8$ & $-123.3(5)$ & $\mathrm{C} 14-\mathrm{S} 2-\mathrm{C} 13-\mathrm{C} 12$ & $123.5(4)$ \\
\hline $\mathrm{C} 1-\mathrm{C} 2-\mathrm{C} 3-\mathrm{Fe} 1$ & $-59.9(3)$ & $\mathrm{Ni} 1-\mathrm{S} 2-\mathrm{C} 13-\mathrm{C} 12$ & $12.2(4)$ \\
\hline $\mathrm{C} 7-\mathrm{C} 2-\mathrm{C} 3-\mathrm{Fe} 1$ & $125.9(5)$ & $\mathrm{C} 14-\mathrm{S} 2-\mathrm{C} 13-\mathrm{C} 20$ & $-62.3(4)$ \\
\hline
\end{tabular}




$\begin{array}{llll}\mathrm{C} 2-\mathrm{C} 3-\mathrm{C} 4-\mathrm{C} 5 & -1.6(5) & \mathrm{N} 11-\mathrm{S} 2-\mathrm{C} 13-\mathrm{C} 20 & -173.6(3) \\ \mathrm{C} 8-\mathrm{C} 3-\mathrm{C} 4-\mathrm{C} 5 & -177.4(4) & \mathrm{C} 13-\mathrm{S} 2-\mathrm{C} 14-\mathrm{C} 15 & -174.9(3) \\ \mathrm{Fe} 1-\mathrm{C} 3-\mathrm{C} 4-\mathrm{C} 5 & 58.2(3) & \mathrm{N} 11-\mathrm{S} 2-\mathrm{C} 14-\mathrm{C} 15 & -65.9(3) \\ \mathrm{C} 2-\mathrm{C} 3-\mathrm{C} 4-\mathrm{C} 9 & 175.9(4) & \mathrm{S} 2-\mathrm{C} 14-\mathrm{C} 15-\mathrm{C} 16 & 72.5(4) \\ \mathrm{C} 8-\mathrm{C} 3-\mathrm{C} 4-\mathrm{C} 9 & 0.1(7) & \mathrm{C} 14-\mathrm{C} 15-\mathrm{C} 16-\mathrm{S} 3 & -73.0(4) \\ \mathrm{Fe} 1-\mathrm{C} 3-\mathrm{C} 4-\mathrm{C} 9 & -124.3(5) & \mathrm{C} 17-\mathrm{S} 3-\mathrm{C} 16-\mathrm{C} 15 & 177.2(3) \\ \mathrm{C} 2-\mathrm{C} 3-\mathrm{C} 4-\mathrm{Fe} 1 & -59.7(3) & \mathrm{N} 11-\mathrm{S} 3-\mathrm{C} 16-\mathrm{C} 15 & 67.5(3) \\ \mathrm{C} 8-\mathrm{C} 3-\mathrm{C} 4-\mathrm{Fe} 1 & 124.4(5) & \mathrm{C} 16-\mathrm{S} 3-\mathrm{C} 17-\mathrm{C} 18 & -122.3(4) \\ \mathrm{C} 3-\mathrm{C} 4-\mathrm{C} 5-\mathrm{C} 1 & 1.6(5) & \mathrm{N} 11-\mathrm{S} 3-\mathrm{C} 17-\mathrm{C} 18 & -10.4(4) \\ \mathrm{C} 9-\mathrm{C} 4-\mathrm{C} 5-\mathrm{C} 1 & -176.0(4) & \mathrm{C} 16-\mathrm{S} 3-\mathrm{C} 17-\mathrm{C} 21 & 60.1(4) \\ \mathrm{Fe} 1-\mathrm{C} 4-\mathrm{C} 5-\mathrm{C} 1 & 60.7(3) & \mathrm{N} 1-\mathrm{S} 3-\mathrm{C} 17-\mathrm{C} 21 & 172.0(3) \\ \mathrm{C} 3-\mathrm{C} 4-\mathrm{C} 5-\mathrm{C} 10 & 173.4(4) & \mathrm{C} 21-\mathrm{C} 17-\mathrm{C} 18-\mathrm{C} 22 & 3.9(7) \\ \mathrm{C} 9-\mathrm{C} 4-\mathrm{C} 5-\mathrm{C} 10 & -4.2(7) & \mathrm{S} 3-\mathrm{C} 17-\mathrm{C} 18-\mathrm{C} 22 & -173.4(3) \\ \mathrm{Fe} 1-\mathrm{C} 4-\mathrm{C} 5-\mathrm{C} 10 & -127.5(5) & \mathrm{C} 21-\mathrm{C} 17-\mathrm{C} 18-\mathrm{S} 4 & 178.1(4) \\ \mathrm{C} 3-\mathrm{C} 4-\mathrm{C} 5-\mathrm{Fe} 1 & -59.1(3) & \mathrm{S} 3-\mathrm{C} 17-\mathrm{C} 18-\mathrm{S} 4 & 0.8(5) \\ \mathrm{C} 9-\mathrm{C} 4-\mathrm{C} 5-\mathrm{Fe} 1 & 123.3(4) & \mathrm{N} 11-\mathrm{S} 4-\mathrm{C} 18-\mathrm{C} 17 & 9.1(4) \\ \mathrm{C} 2-\mathrm{C} 1-\mathrm{C} 5-\mathrm{C} 4 & -1.0(5) & \mathrm{Fe} 1-\mathrm{S} 4-\mathrm{C} 18-\mathrm{C} 17 & 95.5(4) \\ \mathrm{C} 6-\mathrm{C} 1-\mathrm{C} 5-\mathrm{C} 4 & 173.5(4) & \mathrm{N} 1-\mathrm{S} 4-\mathrm{C} 18-\mathrm{C} 22 & -176.3(3) \\ \mathrm{Fe} 1-\mathrm{C} 1-\mathrm{C} 5-\mathrm{C} 4 & -62.3(3) & \mathrm{Fe} 1-\mathrm{S} 4-\mathrm{C} 18-\mathrm{C} 22 & -89.9(3)\end{array}$

Symmetry codes: (i) $-x+2, y,-z+3 / 2$; (ii) $-x+1, y,-z+3 / 2$. 